\title{
Continuous neural activations of simulation and linguistic information during metaphor processing
}

\author{
Pei Liu ${ }^{1}$, Louise Connell ${ }^{1}$ and Dermot Lynott ${ }^{1,2}$ \\ ${ }^{1}$ Department of Psychology, Lancaster University \\ 2 Department of Psychology, Maynooth University
}

\section{Author Note}

We have no conflicts of interest to declare. Correspondence concerning this article should be addressed to Louise Connell (1.connell@lancaster.ac.uk) or Dermot Lynott (dermot.lynott@,mu.ie)

Word count: 7382 


\begin{abstract}
Language processing relies on conceptual representations which are composed of two crucial components, embodied simulation and linguistic distributional pattern. The embodied component refers to the reactivation of previous sensorimotor experiences related to the concept (e.g., experiences with a clever student when reading "bright student"); the linguistic component refers to the co-occurrence pattern of the constituent words (i.e., how often "bright" and "student" appear in the same context). In this study, we examined the existence and roles of these components in metaphor processing. Using both a behavioural study and EEG, we studied how these components affected the speed, success rate and neurophysiological activations of metaphor comprehension. We found that, while performance of metaphor comprehension was mainly influenced by the embodied component, the linguistic component was activated before the embodied component reached its peak and could act as a shortcut to construct good-enough representation, such that people found it easier to accept and hard to reject a metaphor when the distributional frequency of constituent words was high. In other words, the linguistic distributional pattern could provide a guide for conceptual representations before the embodied component was fully engaged.
\end{abstract}

Keywords: Metaphor processing, embodied simulation, linguistic distributional pattern, EEG

Word count: 7382 words 
Continuous neural activations of simulation and linguistic information during metaphor processing

In the past two decades, researchers have gradually come to the consensus that conceptual representation has at least two components, an embodied component and a linguistic component (Barsalou, Santos, Simmons, \& Wilson, 2008; Connell \& Lynott, 2014; Louwerse \& Jeuniaux, 2010; Vigliocco, Meteyard, Andrews, \& Kousta, 2009). As new concepts are acquired, neural networks are activated in response to the physical environment as well as language. The activation of different neural regions leaves traces of conceptual representation; and during conceptual processing (e.g., language comprehension), these traces are reactivated to simulate the sensory, motor, affective, introceptive and other bodily experiences, thus forming the embodied component, as well as to activate the distributional pattern of linguistic symbols, thus forming the linguistic component.

The embodied and linguistic components are highly intercorrelated, because the distributional pattern of words in language often reflects the associations of objects, events, and ideas in the real world (Louwerse, 2011). The fact that bright appears with sun frequently in close proximity reflects the physical reality that the sun is usually bright. Thus, the linguistic information could approximate the embodied component, thereby informing a response on its own; and the linguistic information could in turn activate related simulation information (Johns \& Jones, 2012).

Andrews, Vigliocco, and Vinson (2009) modeled conceptual representation with both simulation and linguistic information and found that word meaning is best represented by the combination of these two types of data. In other words, both embodied and linguistic components are central to the conceptual system. The Language and Situated Simulation theory (LASS: Barsalou et al., 2008) as well as 
the Symbol Interdependency Hypothesis (SIH: Louwerse, 2011) propose that both components are activated simultaneously during language comprehension. The linguistic component reaches the peak of its activation through the distributional patterns, which is relatively faster than the activation of the embodied component and with less cost. The latter, on the other hand, requires deeper processing of situated simulation. As a result, this temporal advantage of the linguistic component could be utilised during conceptual representation depending on the current context or task demands. In other words, conceptual representation is flexible enough to adjust the reliance on the embodied versus the linguistic component in order to satisfy specific task demands (Connell \& Lynott, 2014; Lynott \& Connell, 2010).

Taken together the fact that the linguistic component can provide a fuzzy approximation of the embodied component, and that the former is activated faster than the latter, it was hypothesised that the linguistic component could act as a shortcut to guide conceptual representation before the more costly embodied component is fully engaged, particularly when superficial processing could suffice (Connell, 2018; Connell \& Lynott, 2013). Support for this linguistic shortcut hypothesis should contain two parts. First, the linguistic component should enjoy greater importance during language processing when the task allows relatively shallow and speedy processing. Second, the linguistic component should reach the peak of its activation prior to the embodied component.

Evidence for the former requirement was found with the property verification task and the conceptual combination task. The linguistic distributional pattern among sensory properties (e.g., co-occurrence frequencies between loud and bright) was powerful enough to account for faster responses to these properties, but not for the slower responses (Louwerse \& Connell, 2011). The distributional pattern could also account for responses to novel concepts (e.g., cactus beetle) when people performed a simple yes/no sensibility judgement task instead of an interpretation 
generation task in which they needed to provide a verbal interpretation (Connell \& Lynott, 2013). To support the temporal advantage of the linguistic component, Louwerse and Hutchinson (2012) found in an EEG study that neural regions involved in linguistic processing (e.g., left inferior frontal gyrus) was activated to a larger degree than the regions involved in perceptual processing (near the lingual gyrus) at the early stage of conceptual processing, while the pattern was reversed at the later stage.

In summary, conceptual representation involves both simulation and linguistic information, with the linguistic component acting as a shortcut depending on the demands of the task. As conceptual representation should underlie all types of language processing, we expect the same pattern to be found during metaphor processing as well. In this study, we investigate the interplay between embodied and linguistic components in metaphor processing. Furthermore, we trace their roles during real-time processing using event-related EEG potentials (ERP).

\section{Simulation and Linguistic Representations of Metaphors}

Recent studies have placed metaphor processing under the lens of conceptual representations (Liu, Connell, \& Lynott, 2017, 2018c). In those studies, participants read adjective metaphors (e.g., Student can be bright, Supporters can be lukewarm) that are controlled on both embodied and linguistic components. That is, these metaphors independently varied on two dimensions, which were the ease of simulation (EoS) and the linguistic distributional frequencies (LDF). EoS was a novel norming measure of how much effort it took for people to successfully arrive at a conceptual representation (Liu, Connell, \& Lynott, 2018b), which combined the measures of three scales, that is how easy it was to imagine, to use, and to make sense of the metaphors. The combined EoS measure could outperform any of the individual scale in predicting the performance to a sensibility judgement task. This 
advantage suggested that EoS measured the underlying mechanism of metaphor comprehension, which was assumed to be simulation. On the other hand, LDF was an objective measure of the linguistic distributional patterns, quantified the bi- to 5-gram frequencies of the constituent words (e.g., student and bright with zero, one, two or three intervening words).

Both variables were found to contribute to a unique portion of variance of performance during metaphor processing tasks. People primarily based their performance on the embodied component, as they attempted to form a coherent simulation. They found it easy to accept a metaphor when it was considered easy to simulate, and easy to reject a metaphor when it was considered hard to simulate. The effect of EoS varied based on the depth of processing and the time available for responses. When participants needed to provide an interpretation to the metaphors (i.e., during deeper processing) or when they were allowed more time, EoS showed a larger effect than when they performed a yes/no sensibility judgement (i.e., during relatively shallower processing) or when they were under limited time constraint.

On the other hand, the linguistic component also affected people's judgments. When the constituent words often co-occurred (e.g., bright and student), people could reach a decision to accept the metaphor straightaway, without further expending much effort on simulation. Moreover, information of the linguistic component could guide the activities of embodied simulation. LDF moderated the effect of EoS when people rejected a metaphor as nonsensical (Liu et al., 2018c). When LDF was low, EoS affected the speed of rejection such that the harder it was considered to simulate a metaphor, the faster people were at rejecting it; but when LDF became higher, the effect of EoS diminished and even flipped its direction. That is, for a metaphor which was considered hard to simulate, it took people a long time to reject it as nonsensical if it had a high distributional frequency. What may have happened was that the high LDF suggested that the processing was likely to 
succeed, thus making people spending more effort on simulation, even when processing turned out to be difficult. In a word, evidence supported the linguistic shortcut hypothesis, which suggested that information from the linguistic component served as a guide for further, more costly embodied simulation. A crucial takeaway from these studies is that the embodied and linguistic components were variably engaged based on required depth of processing and the length of response window. These results highlighted the fact that conceptual representation could be highly task-dependent (Connell \& Lynott, 2014; Lebois, Wilson-Mendenhall, \& Barsalou, 2015), and subject to the strategies chosen by participants.

\section{ERP Makers of Metaphor Processing}

As discussed in the previous section, another way to support the linguistic shortcut hypothesis was to study the timecourse of linguistic and embodied components during online processing. It is important to show that the linguistic component in fact reaches the peak of its activations before the embodied component. EEG proves to be a useful tool in this respect because it reveals the real-time activities of the brain during conceptual processing (Amsel, 2011). Previous studies have examined the neural activities during metaphor processing (e.g., Arzouan, Goldstein, \& Faust, 2007; De Grauwe, Swain, \& Holcomb, 2010; Pynte, Besson, Robichon, \& Poli, 1996), but have rarely differentiated the effect of the linguistic component from the effect of the embodied component.

\section{ERP Markers of Simulation versus Linguistic Information}

Liu, Connell, and Lynott (2018a) explored the ERP manifestations of embodied and linguistic components in literal language processing. They found that the neuroelectrical activities associated with the embodied component were different from that of the linguistic component. The former were associated mainly with 
greater activities around 400ms after the stimulus onset in the left parietal region. These activations coincided with the classical N400 effect, which was a marker of greater effort in semantic representation (Kutas \& Federmeier, 2011). In contrast, the linguistic component was activated at first around $300 \mathrm{~ms}$ from the stimulus onset at the frontal region, and again after $600 \mathrm{~ms}$ in the posterior (i.e., parietal and occipital) regions, which might suggest that the linguistic component was associated with the early stage of semantic processing (Collins, Pecher, Zeelenberg, \& Coulson, 2011) as well as conflict resolution (Davenport \& Coulson, 2011; Paczynski \& Kuperberg, 2012) respectively.

\section{Present Study}

In this study, our aim was to replicate and extend previous findings on the linguistic-simulation representations of metaphors. In the behavioural study (Experiment 1), we will replicate the same tasks as previous studies (i.e., the shallow sensibility judgement task, and deep interpretation generation task). Furthermore, to address the possibility that the effect of the tasks in previous studies could be confounded with the differences between participants, we conducted a within-participant design. In this pre-registered experiment (https://aspredicted.org/ff8ms.pdf), we expected to fully replicate the effect of EoS. Also we hypothesised that LDF will behave according to the linguistic shortcut hypothesis, that is LDF will affect the speed of acceptance on its own and guide the activities of embodied simulation, especially when a metaphor was rejected.

Furthermore, we expected both EoS and LDF to be moderated by tasks, such that EoS would have a larger effect in the deep task than the shallow task, and vice versa for LDF.

Then in the EEG study (Experiment 2), we will investigate the continuous neuroelectrical activities of embodied simulation and the activation of linguistic 
distributional patterns. We expect EoS to reach the greatest effect around $400 \mathrm{~ms}$ in the left parietal region, replicating the ERP marker of the embodied component in the behavioural study. In contrast, LDF should have a greater effect early in the trial (around 300ms), thus supporting the linguistic shortcut hypothesis; as well as later after $600 \mathrm{~ms}$ in a similar way as during literal language processing, because metaphor processing should involve the resolution of conflicting representations (Coulson \& Van Petten, 2002). Since these hypotheses were proposed based on the previous study on literal language processing, we expect the results the exceed the current hypothesised regions. As a result, these hypotheses are better considered as tentative, and we will explore the neuroelectrical activities beyond these areas of interest.

\section{Experiment 1: Behavioural Study}

A pre-registered behavioural study here (https://aspredicted.org/ff8ms.pdf) tests the existence and the role of embodied and linguistic information during metaphor processing. In this study, participants performed both a shallow sensibility judgement task and a deep interpretation generation task, which was hypothesised to alter the reliance on the two components. Specifically, the linguistic shortcut hypothesis suggested that shallow processing would encourage the use of the linguistic component. In this section, we report how we determined our sample size, all data exclusions, all manipulations, and all measures in the study.

\section{Method}

Participants. Forty-eight students from Lancaster University participated in the experiment (age: $20.96(S D=3.51)$; female: 31; right-handed: 37$)$. All were native speakers of English, speaking English as their first language since infancy. 
The size of sample was pre-determined to match the previous between-participant study (Liu et al., 2018c).

Materials. 452 metaphors in the form of "NOUN can be ADJECTIVE" (e.g., Student can be bright) were used as stimuli. All adjectives were perceptual adjectives pertaining to vision, audition or haptics (Dantzig, Cowell, Zeelenberg, \& Pecher, 2011; Lynott \& Connell, 2009). Each adjective was paired with four nouns so that the metaphor varied on two dimensions, ease of simulation (EoS) and linguistic distributional frequency (LDF). EoS was operationalised in a norming study (Liu et al., 2018b) which extracted the principle component of three different ratings of the metaphors: sensibility rating (How much sense does the sentence make when you read it in text or hear it in conversation?), usability rating (How easy is it for you to use the sentence in text or conversation?), and imaginability rating (How easy is it for you to imagine the concept described in the sentence?). These three ratings measured the effort to arrive at a mental representation, which, in line with the embodied account of language processing (Barsalou et al., 2008; e.g., Zwaan, 2004,2014 ), is constituted of the simulation of sensorimotor, affective, and other information. Hence, we assumed that the composite measure of these ratings measured the underlying embodied simulation during language comprehension, and thus labeled it ease of simulation (EoS). For instance, Student can be bright $(\mathrm{EoS}=$ $1.84)$ and Solution can be bright $(\mathrm{EoS}=1.41)$ were considered to be easy to simulate; while Illness can be bright $(\mathrm{EoS}=-1.32)$ and Supply can be bright $(\mathrm{EoS}=$ -1.02) were considered to be hard to simulate.

LDF, on the other hand, was defined by the bi- to 5-gram frequencies of each pair of noun and adjective in the Google Web1T Corpus, which had 1 trillion words (Brants \& Franz, 2006). The raw frequency was logarithmically transformed as $\lg (\mathrm{N}$ +41 ), where 41 is the lowest non-zero frequency in the corpus. For example, Student can be bright $(\mathrm{LDF}=4.08)$ and Supply can be bright $(\mathrm{LDF}=3.72)$ had higher 
distributional frequencies than Solution can be bright $(\mathrm{LDF}=3.11)$ and Illness can be bright $(\mathrm{LDF}=2.94)$. The two dimensions (i.e., EoS and LDF) had a correlation of $r=.27$, sharing approximately $7.29 \%$ of variance, and they were separable using statistical measure which will be discussed later in the Results section. The full set of norms can be accessed at https://osf.io/xgysz.

The 452 sentences could be divided into four lists, in which each adjective appeared only once. The EoS and LDF matched across the lists (EoS: $F_{(3,448)}=$ $\left.0.03, p=.99 ; \mathrm{LDF}: F_{(3,448)}=0.09, p=.96\right)$. Thus, two lists of sentences were used for the whole experiment. As the whole experiment was composed of two tasks, participants saw two lists of sentences, one for each task (i.e., each adjective appeared twice throughout the experiment, paired with different nouns).

Procedure. All participants performed two tasks in a counterbalanced order, a sensibility judgement task (or shallow processing condition) and an interpretation generation task (or deep processing condition). Sentences in each task were presented in a random order. Ten extra items were used before each task as practice. In both the practice and the test sessions, participants read the sentences one word/phrase at a time (Figure 1). In the shallow sensibility judgement task, participants were asked to decide whether the sentence was sensible or not. They started by seeing a fixation cross at the beginning of the trial, followed by the noun, "can be", and then the adjective. The adjective stayed on the screen for $200 \mathrm{~ms}$ followed by a blank screen for $2800 \mathrm{~ms}$ during which a response should be made. If they judged the sentence as sensible, participants were asked to press the comma (,) key; and if they judged the sentence as nonsensical, the full stop key (.). Once a response was made, the fixation cross would appear on the screen to signal the beginning of the next trial. However, if no response was made within the length of the response interval, a feedback warning would appear on the screen saying ***TOO $\mathrm{SLOW}^{* * *}$. 
The procedure of the deep interpretation generation task was the same as the shallow sensibility judgement task from the fixation cross until the adjective. After the adjective disappeared, a blank screen remained for 7800ms as a response interval, during which participants were asked to think of an interpretation for the sentence and make a response once they had thought of an interpretation or decided they could not think of an interpretation. The response key was the same as the shallow task. If participants indicated that they had thought of an interpretation for the sentence, after pressing the comma key, a blank screen would appear for them to type down their interpretation of the sentence. They were required not to simply replace the adjective with a synonym. After an interpretation was provided, they would press Enter to proceed to the next trial; and if they indicated that the sentence was not interpretable, they would automatically proceed to the next trial. Like in the shallow task, if no response was made within the length of the response interval, participants would receive a feedback warning. In both tasks, their response decision (accept/reject the metaphor) and the response time (RT) were recorded for analyses. There were two self-administered breaks within each task and one break between tasks.

Design and analysis. The response decisions and RT were analysed as pre-registered. Response decisions (coded 0 for "no" and 1 for "yes") were analysed in a mixed-effect logistic regression. ${ }^{1}$ Task (coded 0 for sensibility judgement and 1

\footnotetext{
${ }^{1}$ We used R (Version 3.5.0; R Core Team, 2017) and the R-packages abind (Version 1.4.5; Plate \& Heiberger, 2016), arm (Version 1.10.1; Gelman \& Su, 2018), BayesFactor (Version 0.9.12.4.2; Morey \& Rouder, 2015), bookdown (Version 0.7; Xie, 2016), broom (Version 0.4.4; Robinson, 2018), coda (Version 0.19.1; Plummer, Best, Cowles, \& Vines, 2006), contrast (Version 0.21; Kuhn, Steve Weston, Wing, Forester, \& Thaler, 2016), cowplot (Version 0.9.2; Wilke, 2017), data.table (Version 1.11.4; Dowle \& Srinivasan, 2017), doBy (Version 4.6.1; Højsgaard \& Halekoh, 2018), dplyr (Version 0.7.5; Wickham, François, Henry, \& Müller, 2018), Formula (Version 1.2.3; Zeileis \& Croissant, 2010), ggplot2 (Version 2.2.1; Wickham, 2009), gridExtra (Version 2.3; Auguie, 2017),
} 


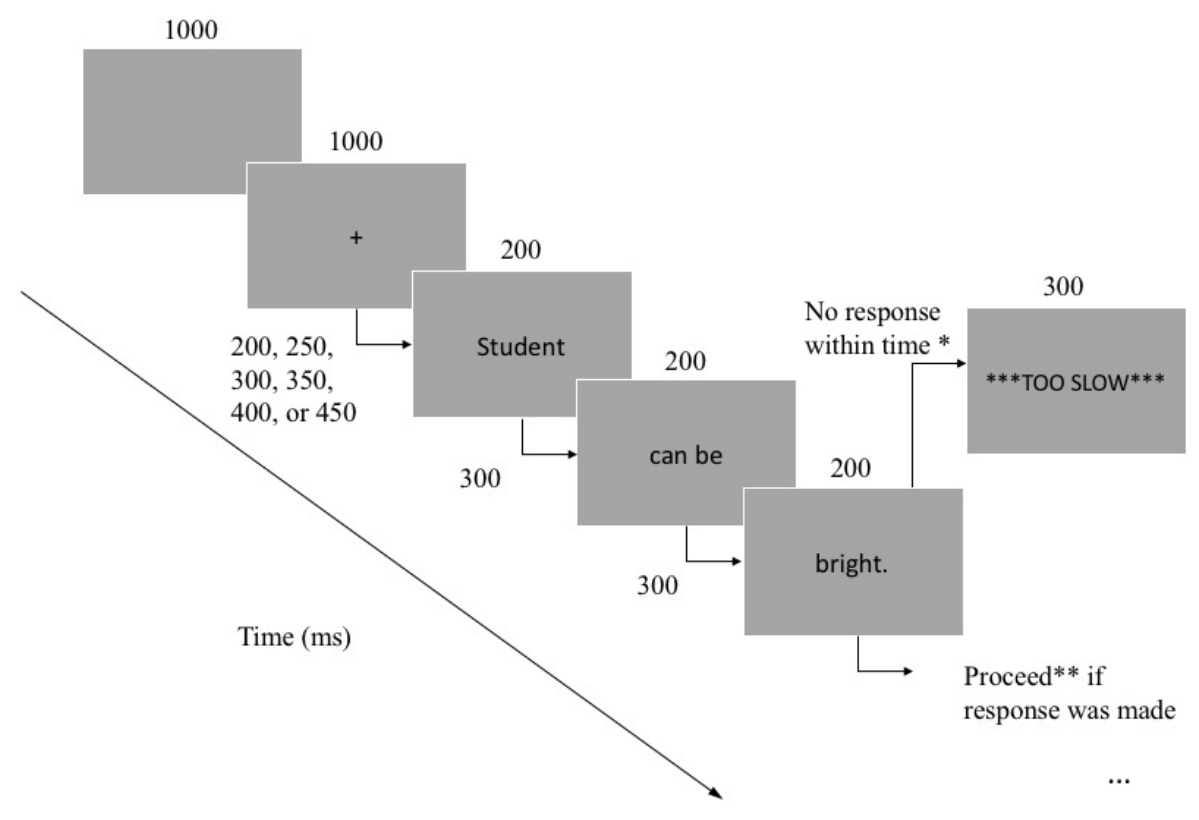

Figure 1. Presentation sequence in Experiment 1. * Response time window depended on task. Shallow sensibility judgment task allowed 3s, and deep interpretation generation task allowed 8s. ** After response, proceed to next trial in shallow sensibility judgment task; and in deep interpretation generation task, proceed to type down the interpretation if answered "yes", and to next trial if answered "no".

for interpretation generation), EoS and LDF, with their interactions, were used as Hmisc (Version 4.1.1; Harrell Jr, Charles Dupont, \& others., 2018), interplot (Version 0.1.5; Solt \& Hu, 2015), knitr (Version 1.20; Xie, 2015), lattice (Version 0.20.35; Sarkar, 2008), lme4 (Version 1.1.17; D. Bates, Mächler, Bolker, \& Walker, 2015), lmerTest (Version 3.0.1; Kuznetsova, Brockhoff, \& Christensen, 2017), magrittr (Version 1.5; Bache \& Wickham, 2014), MASS (Version 7.3.50; Venables \& Ripley, 2002), Matrix (Version 1.2.14; D. Bates \& Maechler, 2017), mgcv (S. N. Wood, 2003, 2004, Version 1.8.23; 2011; S. Wood, N., Pya, \& S"afken, 2016), multcomp (Version 1.4.8; Hothorn, Bretz, \& Westfall, 2008), mvtnorm (Version 1.0.8; Genz \& Bretz, 2009), nlme (Version 3.1.137; Pinheiro, Bates, DebRoy, Sarkar, \& R Core Team, 2017), papaja (Version 0.1.0.9735; Aust \& Barth, 2017), plyr (Wickham, 2011; Version 1.8.4; Wickham et al., 2018), psych (Version 1.8.4; Revelle, 2017), purrr (Version 0.2.5; Henry \& Wickham, 2018), rcartocolor (Version 0.0.22; Nowosad, 2017), rms (Version 5.1.2; Harrell Jr, 2018), SparseM (Version 1.77; Koenker \& Ng, 2017), survival (Version 2.42.3; Terry M. Therneau \& Patricia M. Grambsch, 2000), TH.data 
fixed variables; participant and item were used as crossed random variables modelling random intercepts. ${ }^{2}$ RT was analysed first in an omnibus mixed-effect linear regression, with task, response decision, EoS, LDF and their interactions as fixed predictors; participant and item as crossed random variables modelling random intercepts. $^{3}$

Then RT was separated by the response decision to test the specific hypotheses for different responses, and each analysed in a mixed-effect linear regression. Further analyses of response decisions and RT were conducted in mixed-effect models which modeled random intercepts and slopes (not pre-registered). These analyses were not pre-registered but deemed appropriate because mixed-effect models with random intercepts and slopes would generalise better than a random-intercept-only model (Barr, Levy, Scheepers, \& Tily, 2013). However, a full maximal model did not always converge. Therefore, we looked for the maximal random effect structure justified by the sample for both response decisions and RT data, and reported it here, if they provided a better fit than the random-intercept models. The random-intercept models can be found in Supplementary Materials.

In both the present experiment and Experiment 2, we used the Bayes Factor (Version 1.0.8; Hothorn, 2017), and tidyr (Version 0.8.1; Wickham \& Henry, 2017) for all our analyses and the writing up of this manuscript.

2 Model including participants as random intercepts explained the data better than the empty model by $4.88 \times 10175$ times $\left(B F_{10}=4.88 \times 10^{175}\right)$; model with participants and item as crossed random intercepts further better explained the data than the model with only participants as random intercept $\left(B F_{10}>1.80 \times 10^{308}\right)$; inclusion of fixed predictors further better explained the data by $1.80 \times 10^{132}$ times $\left(B F_{10}=1.80 \times 10^{132}\right)$.

${ }^{3}$ Model including participants as random intercepts explained the data better than the empty model $\left(B F_{10}>1.80 \times 10^{308}\right)$; model with participants and item as crossed random intercepts in fact worsen the model $\left(B F_{10}=0.01\right)$ but was still used for consistency; inclusion of fixed predictors further better explained the data $\left(B F_{10}>1.80 \times 10^{308}\right)$. 
$(\mathrm{BF})$, instead of the Chi-squared test, to decide whether the addition of new random or fixed variables improved a model. BF uses the Bayesian approach to calculate the amount of evidence the data lends to the alternative hypothesis in relations to the amount of evidence for the null hypothesis. $\mathrm{BF}_{10}$ denotes the ratio of the likelihood of the data under the alternative hypothesis against the null hypothesis. If $\mathrm{BF}_{10}$ is 5 , it means that the data is 5 times more likely to occur under $\mathrm{H}_{1}$ than $\mathrm{H}_{0}$. Therefore, BF can inform us about quality of the hypotheses. We can be more confident with the alternative hypothesis if $B F_{10}=100$ than if $B F_{10}=10$. Inversely, if $\mathrm{BF}_{10}$ is less than 1 , we would be more confident about the null hypothesis than the alternative hypothesis. As a result, the $\mathrm{BF}$ is superior to $p$-values produced by null-hypothesis significance testing (NHST), which only tells us the probability of Type I error but not how good the alternative hypothesis is. See Jarosz and Wiley (2014) and Wagenmakers (2007) for detailed discussions.

The $\mathrm{BF}$ of regression models can be easily obtained in $\mathrm{R}$ by extracting the Bayesian information criteria $(\mathrm{BIC}) . \mathrm{BF}_{01}$ (comparing $\mathrm{H}_{0}$ against $\mathrm{H}_{1}$ ) is calculated as:

$$
B F_{01}=e^{\frac{\left(B I C\left(H_{1}\right)-B I C\left(H_{0}\right)\right)}{2}}
$$

$\mathrm{BF}_{10}$, which denotes the likelihood of $\mathrm{H}_{1}$ against $\mathrm{H}_{0}$ is simply the inverse of $\mathrm{BF}_{01}$. While the continuous scale of $\mathrm{BF}$ is useful enough, we could conveniently describe the results, using Jeffreys (1998)'s guideline to characterise BF (Table 1).

\section{Results and Discussion}

No participants were deleted for slow response ( $>$ 3SD from grand mean). $1.35 \%$ of trials were missed (i.e., no response within the time limit), which were deleted. Eight trials were deleted for motor error (RT $<200 \mathrm{~ms})$. Beyond that, 39 
Table 1

Interpretation of $B F$ as evidence

for $H 1$

\begin{tabular}{ccc}
\hline$B F_{01}$ & $B F_{10}$ & Support for $H_{1}$ \\
\hline $1-.33$ & $1-3$ & Anecdotal \\
$.33-.10$ & $3-10$ & Substantial \\
$.10-.03$ & $10-30$ & Strong \\
$.03-.01$ & $30-100$ & Very strong \\
$<.01$ & $>100$ & Decisive \\
\hline
\end{tabular}

trials in the deep processing task were marked out as invalid interpretations (blank, "0", "wrong button", etc.). Finally, we removed individual trials with outlying RT ( $>$ 3SD from individual means per response per task) that was $0.80 \%$ and $1.34 \%$ of "yes" and "no" responses respectively in the shallow task; $1.05 \%$ and $1.69 \%$ of "yes" and "no" response respectively in the deep task. Consequently, there remained 5294 data points for the shallow task and 5199 for the deep task.

Response decision. The general acceptance rate was $46.07 \%$. That was $47.02 \%$ of the shallow task and $45.10 \%$ of the deep task. Mixed-effect logistic regressions were conducted. The random-intercepts model as per pre-registration showed different results from the maximal random-slopes model justified by the data, but the latter explained the data better than the former $\left(B F_{10}=8.57 \times 10^{32}\right.$; detailed explanation of BF in Experiment 2). Therefore, we reported the maximal model here (Table 2). The results showed that only EoS, among all fixed predictors, had a significant effect on response decisions. As EoS increased by one unit, the odds to accept the metaphors increased 3.75 times. The random-intercepts model showed that beyond the effect of EoS, EoS interacted with task and LDF also had a 
Table 2

Logistic mixed-effect regression of response decision

\begin{tabular}{llc}
\hline & $b$ & $95 \% \mathrm{CI}$ \\
\hline Intercept & $-0.28 \dagger$ & $-0.58-0.02$ \\
Task & -0.15 & $-0.39-0.1$ \\
LDF & 0.07 & $-0.01-0.15$ \\
EoS & $1.32 * * *$ & $1.16-1.48$ \\
Task : LDF & 0.05 & $-0.03-0.13$ \\
Task : EoS & 0.01 & $-0.08-0.1$ \\
LDF : EoS & 0.05 & $-0.02-0.13$ \\
Task : LDF : EoS & -0.01 & $-0.1-0.07$ \\
\hline
\end{tabular}

Note. $\dagger$ means $p<.10 ;{ }^{*}$ means $p<.05$;

** means $p<.01 ; * * *$ means $p<.001$.

$b$ is non-standardised regression coefficient.

positive effect on response decision (see Supplementary Materials).

Response time. The grand mean of RT was $1391 \mathrm{~ms}(S D=885 \mathrm{~ms})$, which was $1114 \mathrm{~ms}(S D=455 \mathrm{~ms})$ for shallow task and $1674 \mathrm{~ms}(S D=1102 \mathrm{~ms})$ for deep task. The average RT per response decision per task was shown in Table 3.

Analyses of RT found evidence of net suppression (J. Cohen, Cohen, West, \& Aiken, 2003), which means that the shared variance of LDF and EoS was masking the true relationship between the variables and RT. Because LDF and EoS correlated to some degree, LDF contributed to the fit of the model by explaining the error variance of EoS. As a result, the effect of EoS was enhanced while the effect of LDF was diminished. This could be seen by the fact that the effect of LDF in the 
Table 3

\begin{tabular}{lcc} 
Mean (SD) $R T$ in ms per task per \\
response decision \\
\hline Task & Shallow & Deep \\
\hline Yes & $1118(457)$ & $1815(1085)$ \\
No & $1110(454)$ & $1557(1102)$ \\
\hline
\end{tabular}

LME model was the opposite of its zero-order correlation with RT. As seen in Supplementary Materials, LDF had a negative effect on the speed to reject a metaphor (i.e., "no" RT) in the mixed-effect model, but a positive correlation with RT according to the zero-order correlations. Therefore, as pre-registered, we orthogonalised EoS and LDF using a principle components analysis (PCA) with varimax rotation and Kaissar covariance normalisation. PCA managed to obtain two perpendicular variables each corresponding to one of the original variables. This procedure solved the problem of net suppression because the orthogonal variables did not have any shared variance. The orthogonal variables obtained by PCA did not lose any information from the original variables, and they correlated with each variable $(r=.99)$. The orthogonalisation procedure was done both for the omnibus regression and separately with the RT to "yes" and "no" responses for the analyses per response. The results reported here used only orthogonal variables.

The omnibus regression was shown in Table 4. Again, the maximal model justified by the data provided a massively better fit than the random-intercept model $\left(B F_{10}>1.80 \times 10^{3084}\right)$, so the former was reported here and the latter in Supplementary Materials. EoS had a positive effect when LDF was at its mean, which was moderated by task and response. It showed that EoS had a positive effect

\footnotetext{
${ }^{4}$ The largest number calculable on a computer is $2^{1024}$, which is approximately $1.80 \times 10^{308}$.
} 
Table 4

Omnibus linear mixed-effect regression of $R T$

\begin{tabular}{lrr}
\hline & $b$ & \multicolumn{1}{c}{$95 \%$ CI } \\
\hline Intercept & $1424.55 * * *$ & $1293.91-1555.2$ \\
Task & $411.02 * * *$ & $283.14-538.89$ \\
Response & 28.53 & $-1.2-58.25$ \\
LDF & 2.4 & $-17.76-22.56$ \\
EoS & $58.51 * * *$ & $37.49-79.53$ \\
Task : Response & 19.4 & $-22.48-61.28$ \\
Task : LDF & 15.82 & $-11.64-43.29$ \\
Response : LDF & -10 & $-37.86-17.86$ \\
Task : EoS & $49.02 * * *$ & $20.53-77.51$ \\
Response : EoS & $-189.33 * * *$ & $-217.94--160.71$ \\
LDF : EoS & $-31.84 * *$ & $-50.88--12.81$ \\
Task : Response : LDF & -16.08 & $-55.36-23.21$ \\
Task : Response : EoS & $-101.39 * * *$ & $-141.15--61.64$ \\
Task : LDF : EoS & -6.27 & $-32.04-19.51$ \\
Response : LDF : EoS & 22.49. & $-3.91-48.89$ \\
Task : Response : LDF : EoS & 4.6 & $-32.04-41.24$ \\
\hline
\end{tabular}

Note. $\dagger$ means $p<.10{ }^{*}$ means $p<.05$;

** means $p<.01 ; * * *$ means $p<.001$.

$b$ is non-standardised regression coefficient. 
for both tasks, which was larger for the deep task than the shallow task. The effect was positive for "yes" response but negative for "no" response. In contrast, LDF did not have an effect when EoS was at its mean regardless of task and response. However, LDF did interact with EoS when response was "no" in the sensibility judgement task, which was attenuated by "yes" response and the deep interpretation generation task, as will be discussed in separate models below.

Because of the interaction between response decision and other variables, and our hypotheses that opposite effects would exist for "yes" and "no" responses, we separated the data into two sets and analysed the RT per response with re-orthogonalised variables (Table 5). For "yes" response (i.e. when participants accepted the metaphors), both EoS and LDF had a negative effect on both tasks. That is as EoS or LDF increased, people were faster at accepting a metaphor. Only EoS interacted with task, which means that it had a larger (more negative) effect when people needed to generate an interpretation for the metaphor than when they needed to make a simple "yes/no" judgement concerning sensibility; whereas the interaction between LDF and task was not significant meaning that the effect of LDF did not change between tasks.

For "no" response (i.e. when people rejected the metaphors), EoS had a significant, positive effect when LDF was at its mean, that is as ease of simulation increased, it took people longer to reject a metaphor. This effect of EoS interacted with task as well, such that EoS had a larger (more positive) effect for the deep task than the shallow task. LDF further interacted with EoS with a negative term, such that as LDF increased, the effect of EoS decreased. This interaction remained the same for both shallow and deep processing (Figure 2).

Summary. EoS strongly affected metaphor processing as predicted. As metaphors become easier to simulate, the likelihood and speed of accepting them increased regardless of LDF; whereas the speed of rejecting them decreased, which 
Table 5

Mixed-effect linear regression model of $R T$ per response decision

\begin{tabular}{lrrrrr}
\hline & \multicolumn{2}{c}{ Yes } & \multicolumn{2}{c}{ No } \\
\cline { 2 - 3 } \cline { 5 - 5 } & $1465.66 * * *$ & $1336.17-1595.14$ & $1457.7 * * *$ & $1304.26-1611.13$ \\
\hline Intercept & $476.63 * * *$ & $345.85-607.42$ & $431.55 * * *$ & $280.32-582.78$ \\
Task & $-25.03 *$ & $-45.81--4.25$ & 14.8 & $-5.35-34.95$ \\
LDF & $-158.14 * * *$ & $-191.16--125.13$ & $67.32 * * *$ & $35.1-99.54$ \\
EoS & -9.64 & $-39.56-20.29$ & 17.48 & $-8.74-43.69$ \\
Task : LDF & $-71.75 * * *$ & $-102.57--40.93$ & $41.21 * *$ & $14.55-67.87$ \\
Task : EoS & -8.14 & $-27.8-11.53$ & $-27.29 * *$ & $-44.89--9.69$ \\
LDF : EoS & -3.78 & $-32.27-24.71$ & -5.48 & $-29.18-18.22$ \\
Task : LDF : EoS & & & & \\
\hline
\end{tabular}

Note. $\dagger$ means $p<.10{ }^{*}$ means $p<.05$,

** means $p<.01 ; * * *$ means $p<.001$.

$b$ is non-standardised regression coefficient.

'Yes' RT maximal model over random-intercepts model: $B F_{10}=2.80 \times 10^{194}$.

'No' RT maximal model over random-intercepts model: $B F_{10}>1.80 x 10^{308}$ 


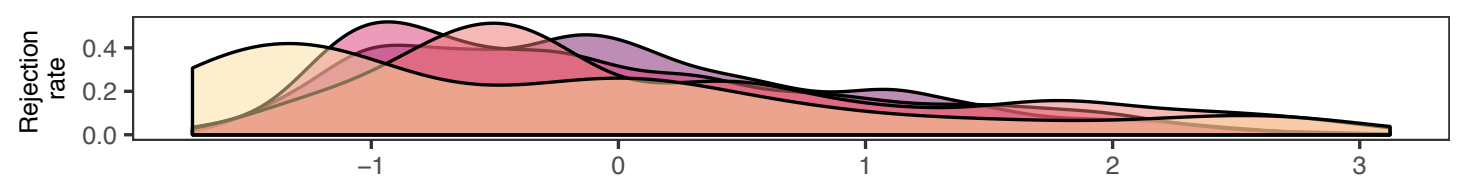

Quartile of LDF $\square$ 1st $(-1.42,-0.292] \square$ 2nd $(-0.292,0.832] \square$ 3rd $(0.832,1.96] \square$ 4th $\square(1.96,3.08]$

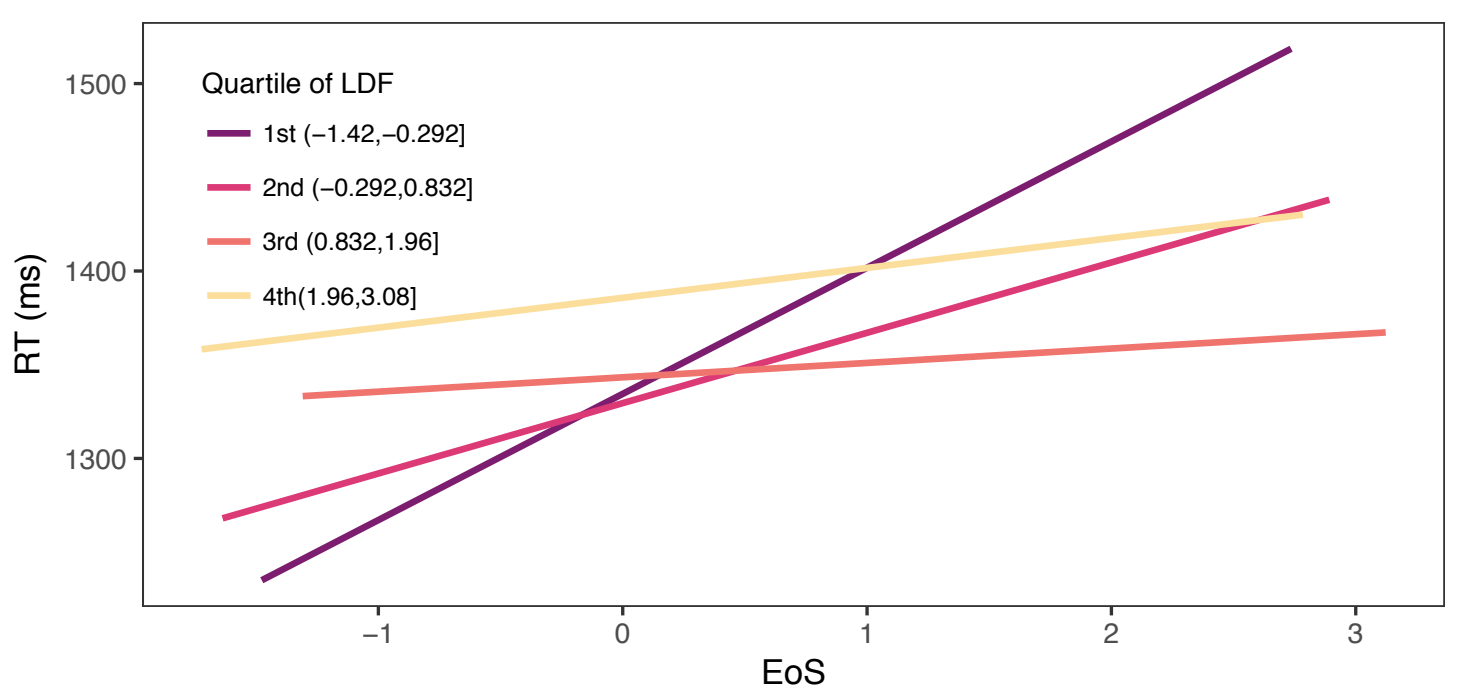

Figure 2. Effects of EoS on the "no" RT at four quartiles of LDF. As LDF increased from the 1st quartile (violet) to the highest 4th (yellow), the effect of EoS flatlined. The density plot on the top shows the proportion of "no" response along EoS per quartile of EoS.

was attenuated when LDF increased. That is to say, when the constituent words did not often co-occur, the easier it was to simulate a metaphor, the harder it was to reject it; but when constituent words often co-occurred, the effect of EoS decreased and flatlined.

LDF, though not affecting response decisions, had a significant effect on RT as expected, such that as constituent words co-occurred more often, the speed of accepting metaphors became faster. LDF also had an effect on the rejection speed as expected, but the effect was only limited to low EoS. In other words, when a metaphor was hard to simulate, the more often the constituent words co-occurred, 
the slower it was for people to reject it; but when a metaphor was easy to simulate, the effect disappeared. As a result, it was fastest to reject a metaphor when both EoS and LDF were low, and slowest when LDF was low but EoS was high.

Task was found to moderate EoS as expected. The effect of EoS was larger for the deep interpretation generation task than for the shallow sensibility judgement task. In contrast, the effect of LDF was not affected by task, contrary to our hypotheses. The negative effect of LDF on the acceptance speed remained the same for both sensibility judgement and interpretation generation task, and the interaction between EoS and LDF on the rejection speed did not vary between tasks either.

\section{Experiment 2: EEG Study}

In this experiment, we will examine the neuroelectrical activations of embodied and linguistic components during metaphor processing. As this study was the first to the authors knowledge to study the simulation-linguistic representation of metaphor processing, we did not have any confirmatory hypotheses concerning the results. However, we proposed some tentative hypotheses concerning the timecourse and scalp regions of the activations. The activations of the embodied component will be manifested around $400 \mathrm{~ms}$ after the adjective onset at the left parietal region. The activations of the linguistic component will be prior to those of the embodied component, possibly around $300 \mathrm{~ms}$ in the frontal region; and they will re-emerge after $600 \mathrm{~ms}$ in the parietal region. Although the experiment was not pre-registered, we will report here how we determined our sample size, all data exclusions, all manipulations, and all measures in the study.

\section{Method}


Participant. Sixteen native speakers of English were needed to match previous studies on metaphor processing (e.g., Coulson \& Van Petten, 2002) and the property verification task of (Liu et al., 2018a). One participant was excluded because the data was not recorded properly due to technical trouble, so one more was recruited to make up for the loss. All resulting usable participants were students of Lancaster University (age: $22.06(S D=6.13)$; female: 11; right-handed: 15$)$.

Materials. The stimuli derived from the same set of metaphoric sentences as ones in Experiment 1. The only difference was that the sentences in this experiment did not end after the adjectives, but all had the word "sometimes." as the end, in order to prevent the end of sentence wrap-up effect (Brouwer, Fitz, \& Hoeks, 2012; Kutas, Federmeier, \& Sereno, 1999), which could be more noisy because it took longer time and involved the integration of phrases and clauses and the processing of phrase boundaries. The sentences were distributed into six lists of 169 or 170 items, each composted of one and a half lists of items from Experiment 1. In each of these six lists in this experiment, half of the adjectives appeared twice pairing with two different nouns and half appeared only once.

Procedure. The items lists were used both in the sensibility judgement task and in the interpretation generation task. Participants took both tasks each with a different list, such that they saw different sentences (different nouns, same adjectives) between the two tasks. The order of the tasks were counterbalanced and the order of sentences were fully randomised. Participants were given four self-administered break in each task as well as one long break between tasks, resulting in five blocks of approximately 34 trials in each task.

At the beginning of each block, participants were given a four-second waiting period for the EEG recording to initiate. The trial structure was similar to Experiment 1 with few changes (Figure 3). Before the fixation cross, we added 1 second of blinking time during which a cue to blink remained on the screen. A 


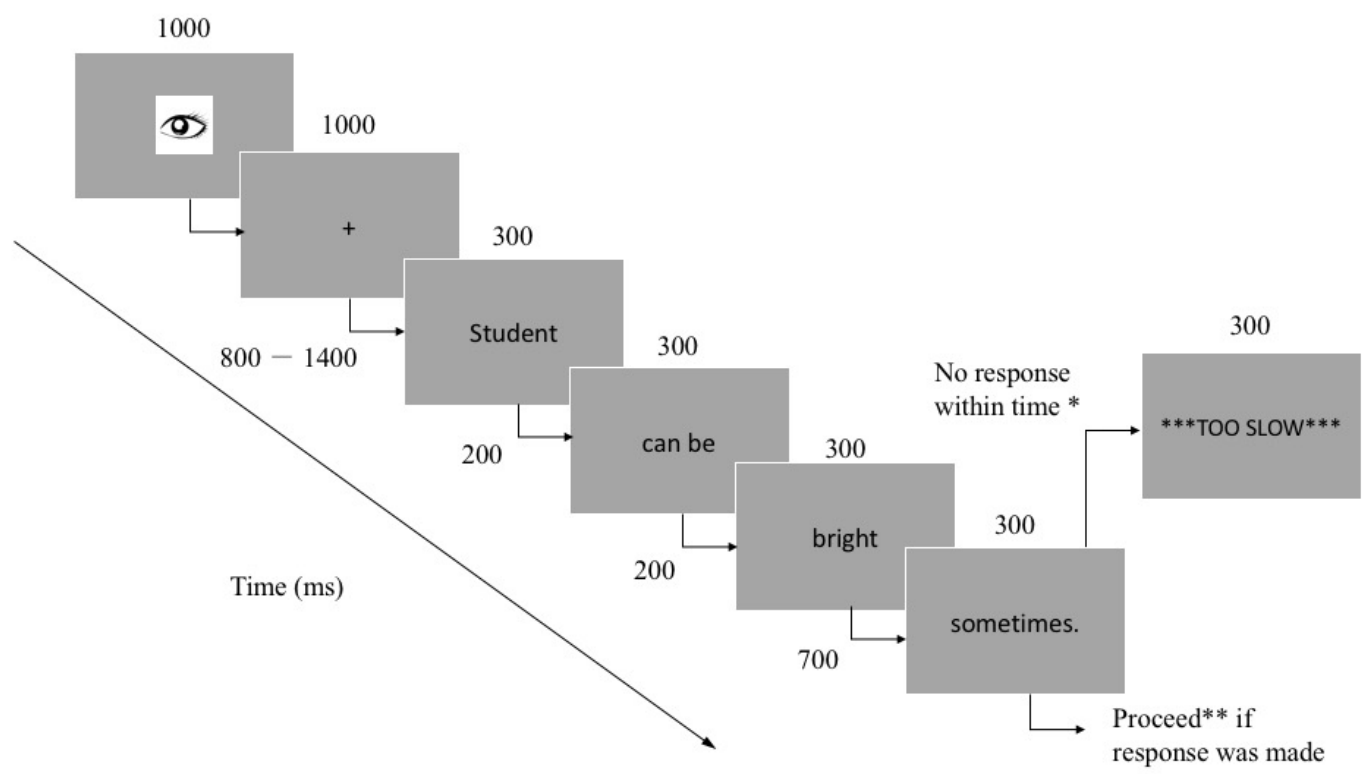

Figure 3. Presentation sequence in Experiment 2. ${ }^{*}$ Response time window depended on task. Shallow sensibility judgment task allowed 3s, and deep interpretation generation task allowed $8 \mathrm{~s}$ (including the word). ${ }^{* *}$ After response, proceed to next trial in shallow sensibility judgment task; and in deep interpretation generation task, proceed to say out loud the interpretation if answered "yes", and to next trial if answered "no".

fixation cross followed for a variable amount of time between $800 \mathrm{~ms}$ to $1400 \mathrm{~ms}$. Then, the nouns, "can be", and the adjectives word were presented sequentially. After the adjectives disappeared, a blank screen remained for 700ms during which participants did nothing but focusing on the screen, in order to eliminate the readiness potential which would occur if participants needed to make a response immediately after the adjectives (Dehaene et al., 1998). Following the blank screen after the adjective, the word "sometimes" would follow for another 300ms as the end of the sentence followed by a blank screen as the response interval (2700s for the shallow task and $7700 \mathrm{~ms}$ for the deep task), during which participants were required to perform either the sensibility judgement task or the interpretation generation task as Experiment 1. 
To make a response, participants were asked to press a red button at the position of " $\mathrm{F}$ " on the keyboard for "yes", or a white button at the position of "J" for "no". In the sensibility judgement task, the experiment will proceed to the next trial after the button press, but in the interpretation generation task, if participants pressed "yes", they would see a sign of a microphone on the screen and they would say their interpretation of the sentence out loud. Their interpretations were recorded by an external Sony recorder. This was done differently from Experiment 1 to avoid contamination of the data by unwanted head movements during typing. After the interpretation was recorded, they pressed the space bar to move on to the next trial. Like in Experiment 1, if no response was made when the response interval had elapsed, a feedback warning was shown on the screen.

Apparatus. Words were presented on a 19-inch CRT monitor operating at $100 \mathrm{~Hz}$ refresh rate using EPrime 1.0, and behavioural responses were recorded using a QWERTY keyboard. High-density EEG was recorded continuously using Hydrocel Geodesic Sensor Nets (Electrical Geodesics Inc., Eugene, OR, USA) at 128 locations referenced to the vertex $(\mathrm{Cz})$ online. The ground electrode was at the rear of the head (between $\mathrm{Cz}$ and $\mathrm{Pz}$ ). Electrophysiological signals were acquired at the sampling rate of $1000 \mathrm{~Hz}$ by an Electrical Geodesics Inc. amplifier with a band-pass filter of $0.1-100 \mathrm{~Hz}$. The impedance was checked prior to the beginning of the recording and they were considered acceptable if lower than $50 \mathrm{k} \Omega$.

EEG analysis. The digitized EEG was further processed offline using NetStation v 4.5.4. It was band-pass filtered between $0.1-30 \mathrm{~Hz}$ and was segmented into epochs including $200 \mathrm{~ms}$ before adjective onset and 1000ms after adjective onset. EEG epochs were automatically rejected for body and eye movements the signal exceeded $\pm 200 \mu \mathrm{V}$ at any electrode. Data were further checked through visual inspection for eye-movements, eye-blinks and other body movement artifacts not detected by the automated algorithm. The artifact free segments were 
baseline-corrected with respect to the average amplitude in the $200 \mathrm{~ms}$ window proceeding stimulus onset, and were re-referenced to the average reference offline.

These segments were then exported to a comma-separated values (csv) file and analysed in R. In order to explore the continuous activations of embodied and linguistic components, we analysed the EEG output throughout the whole 1-second epoch after the adjective onset, and across the whole scalp (Amsel, 2011; Hauk, Pulvermüller, Ford, Marslen-Wilson, \& Davis, 2009; Hendrix, Bolger, \& Baayen, 2017; Liu et al., 2018a). The EEG output per participant per stimulus were sliced into 20 segments of $50 \mathrm{~ms}$ and were averaged. The electrodes analysed were the 90 electrodes in the area on the scalp covered by the 20-20 EEG net (Appendix B). The resulting datapoints were analysed in linear mixed-effect models (LME) with participants and items as crossed random variables to predict random intercepts. The analyses were exceptionally computationally heavy and thus were carried out distantly using the High End Computing Cluster (HEC; http://www.lancaster.ac.uk/iss/services/hec/) of Lancaster University.

Using LME offers two advantages over the conventional method of analysis using ANOVA. First, it solves the language-as-fixed-effect fallacy (Clark, 1973) by accounting for the random variance from both participants and stimulus at the same time. Second, LME offers the possibility to take on continuous variables. In the present study, both critical variables (i.e., EoS and LDF) are continuous. Forcing them into distinct categories (e.g., high frequency vs low frequency) causes the loss of data, and may mask the true relationship between the predictor and the dependent variable (Amsel, 2011; Baayen, Davidson, \& Bates, 2008). In a word, using LME to treat EEG data can avoid the inflation of Type I error rate and provide greater power.

Because of the behavioural results from Experiment 1, we have reason to believe that EoS and LDF will have different effects on "yes" and "no" response. 
Therefore, in the analysis of ERP, we treated the key variables in separate models per response decision. We created LME with task (deep interpretation generation coded as 0 ; shallow sensibility judgement coded as 1) and the critical variables. Following Experiment 1, we also orthogonalised EoS and LDF per response. In order to ascertain evidence in favour of (or against) each key variable, we conducted model comparison in a hierarchical fashion. The baseline model was composed of participants and items as crossed random variables modelling random intercepts, and no fixed effects. Then in each hierarchical step, new fixed effects are added in and the new model was compared to the model in the previous step.

For the effects of EoS, the models were built in the following three steps.

Step 1: Baseline model + task as a fixed effect;

Step 2: Step 1 model + EoS as a fixed effect;

Step 3: Step 2 model + Interaction between task and EoS as fixed effect.

For the effects of LDF, the same three steps as above were repeated.

Two further model comparisons were conducted to extract the relative advantages of EoS and LDF:

1. Comparison between the EoS model obtained from Step 2 (i.e., EoS + task as fixed effects) with the LDF model obtained from Step 2;

2. Comparison between the EoS model obtained from Step 3 with the LDF model obtained from Step 3.

We again used BF to conduct these model comparisons. Apart from the superiority over $p$-values as discussed in Experiment 1, BF further offered a practical advantage specific to this experiment, that is $\mathrm{BF}$ does not require post hoc corrections of multiple comparisons. In this experiment, we need to conduct multiple analyses to the same set of data, which dramatically inflates the Type I 
error rate. Five models need to be built per time slice per electrode, resulting in 9000 models in total. Although a post hoc correction can be conducted (e.g., Bonferroni correction), it will render the test unnecessarily conservative. To keep the family-wise critical level as .05, a critical level of $5.56 \times 10^{-6}$ is needed per model. This stringent criterion would render any $p$-values we can obtain meaningless. As a result, we chose to report only $\mathrm{BF}$ in this experiment together with the regression coefficients from LME models for effect size (results of NHST can be found in Supplementary Materials).

\section{Results}

Data cleaning. The data was cleaned in a similar way as Experiment 1. No participants were removed for slow responses. $0.98 \%$ of trials were missed and 3 trials in the interpretation generation task were removed due to invalid interpretations. No trials were removed because of motor error because by the time a response was made after the last word of the sentence, participants already had $1 \mathrm{~s}$ to process the sentence. 5537 trials remained for the analysis, which included 2753 trials for the deep task and 2784 for the shallow task.

Behavioural results. Analyses of the behavioural data were conducted with participants and items as crossed random factors modelling random intercepts and slopes in a maximal model justified by the data. The response decision was analysed in a logistic mixed-effect regression ${ }^{5}$ and RT was analysed in linear

\footnotetext{
${ }^{5}$ Model including participants as random intercepts explained the data better than the empty model by $7.87 \times 10^{123}$ times $\left(B F_{10}=7.87 \times 10^{123}\right)$; model with participants and item as crossed random intercepts further better explained the data than the model with only participants as random intercept by $7.68 \times 10^{174}$ times $\left(B F_{10}=7.68 \times 10^{174}\right)$; inclusion of fixed predictors further better explained the data by $3.21 \times 10^{131}$ times $\left(B F_{10}=3.21 \times 10^{131}\right)$.
} 
mixed-effect regressions ${ }^{6}$ as Experiment 1. The results were largely similar to those of Experiment 1 (see Supplementary Materials), with differences in the effect of LDF on rejection speed. First as predicted, LDF had a positive effect on rejection speed when EoS was at the mean in this experiment $(b=26.54,95 \% \mathrm{CI}=[10.96,42.12]$, $\mathrm{df}=2817.30, \mathrm{t}=3.34, p<.001)$. Second, it also interacted with EoS, such that as LDF increased, the effect of EoS also increased $(b=19.32,95 \% \mathrm{CI}=[4.63,34.00]$, $\mathrm{df}=2817.60, \mathrm{t}=2.579, p=.010)$. Again, the effect of EoS increased in deep processing task, while there was no evidence that the effect of LDF increased in shallow processing task.

\section{ERP results.}

\section{Overview.}

The timecourse of the critical variables was illustrated by the mean BF by comparing EoS models with LDF models in Step 2 and Step 3 (Figure 4; all BFs are in the Supplementary Materials). Strong evidence supported the activations of EoS from 400ms and persisted until the end of the epoch for "yes" response. Two peaks of activations were first around $500 \mathrm{~ms}$, and then after $750 \mathrm{~ms}$. No strong evidence for $\mathrm{LDF}$ was found overall, that is no mean BF was below 0.1. At two points LDF seemed to have slightly outperformed EoS (i.e., $B F_{E L}<1$ ), which were at $200 \mathrm{~ms}$ when comparing the Step 2 models and at 600ms when comparing the Step 3 models. Further investigations into the topography of the key variables will show localised activations of the key variables in more details.

\section{Task effects.}

\footnotetext{
${ }^{6}$ Model including participants as random intercepts explained the data better than the empty model by $1.40 \times 10^{117}$ times $\left(B F_{10}=1.40 \times 10^{117}\right)$; model with participants and item as crossed random intercepts in fact worsen the model $\left(B F_{10}=0.01\right)$ but was still used for consistency; inclusion of fixed predictors further better explained the data by $1.01 \times 10^{72}$ times $\left(B F_{10}=1.01 \mathrm{x}\right.$ $\left.10^{72}\right)$.
} 


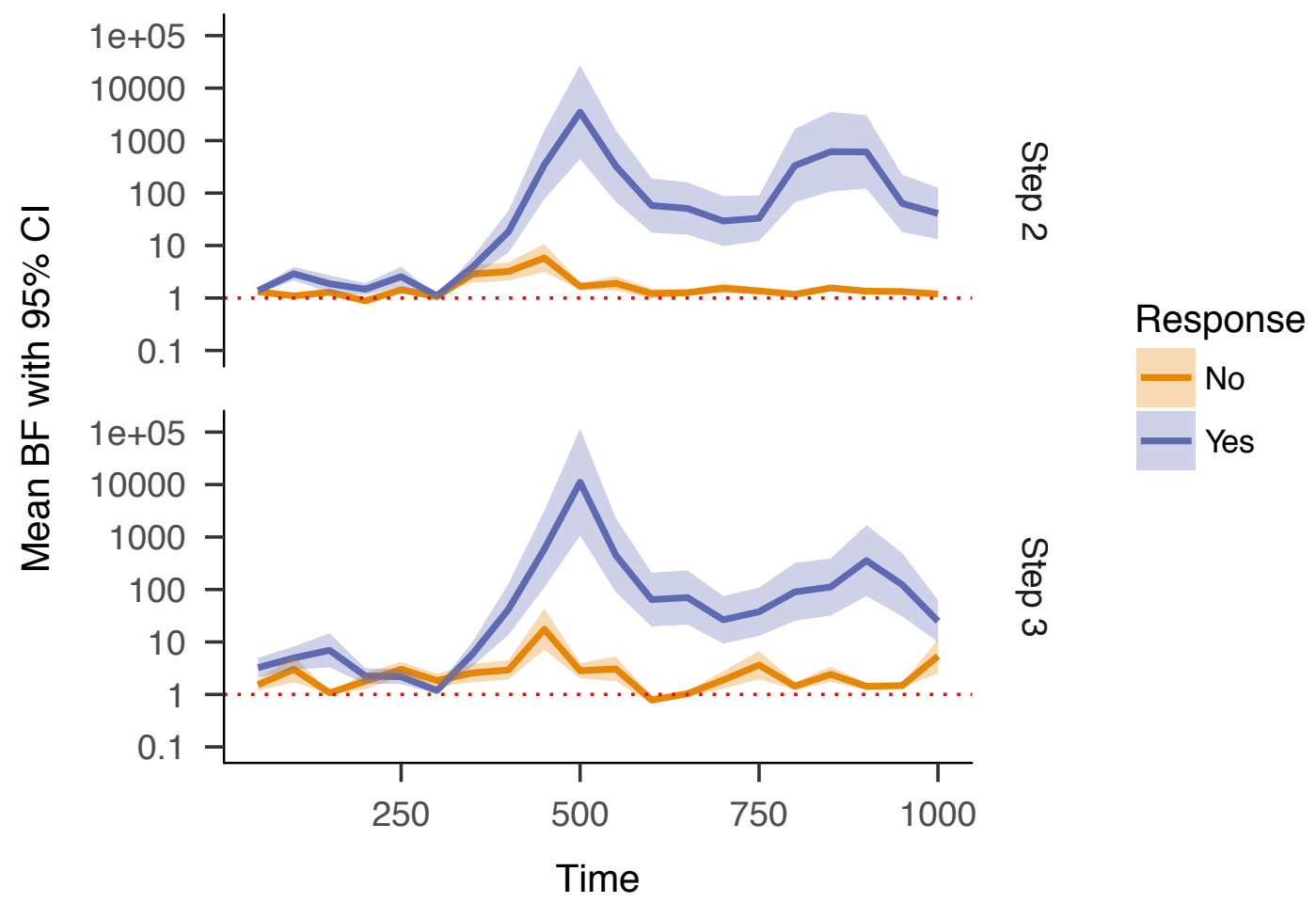

Figure 4. Logarithmically transformed mean $B F_{E L}$ of models of EoS compared with models of LDF in Step 2 (which excluded the interaction terms) and Step 3 (which included the interaction term) with $95 \%$ CI. The red dotted line is the cutoff when $B F_{E L}=1$ (i.e., EoS outperformed LDF when above the line, and LDF outperformed EoS below the line).

Step 1 (Figure 5) of model comparison showed strong evidence that task had an effect on EEG amplitude. For "yes" response, the effect started from 400ms after onset and lasted until the end of the epoch, peaking around 700ms; for "no" response, the effect only appeared around $650 \mathrm{~ms}$. In the following steps, we obtained evidence for EoS and LDF separately, above and beyond the effect of task.

\section{EoS effects.}

"Yes" response.

Our hypothesis for EoS stated that we should find strong effect of EoS in the 


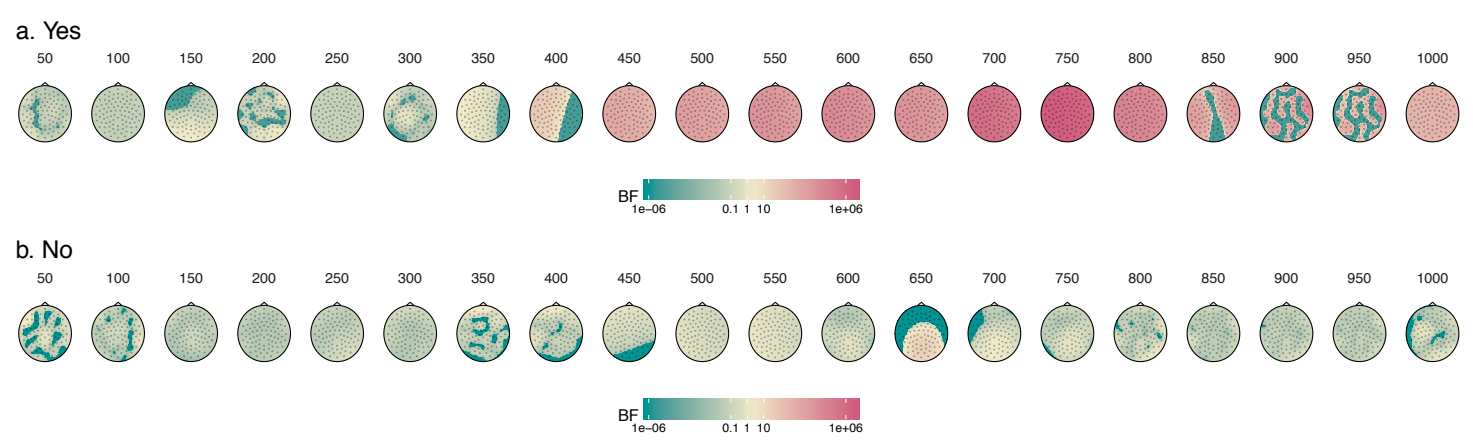

Figure 5. Task effect for a. "yes" response, and b. "no" response. When $B F_{10}>10$, the data strongly supported the effect of task over the baseline model; whereas when $B F_{10}$, the data strong supported the baseline model over the effect of task. BF was logarithmically transformed for plotting.

left parietal area round 400ms (similar to a typical N400 effect). This was confirmed by Step 2 of model comparison for "yes" response, which showed evidence for EoS from $400 \mathrm{~ms}$ to $600 \mathrm{~ms}$, when people accepted the metaphors (Figure 6 a and c). The data were on average 6.41 times (i.e., mean $B F_{21}=6.41$ ) and 148.54 times maximally $\left(\max B F_{21}=148.54\right)$ more likely to occur in models including EoS than the task-only models excluding EoS. EoS had a positive effect on amplitude, that is to say: metaphors that were harder to simulate elicited a more negative amplitude. From Panel c, it could be seen that in the left parietal region the effect was most prominent near the central line (e.g., E37: mean $b$ between 400ms and 600ms was 0.56 , mean $\left.B F_{21}=66.75\right)$. 

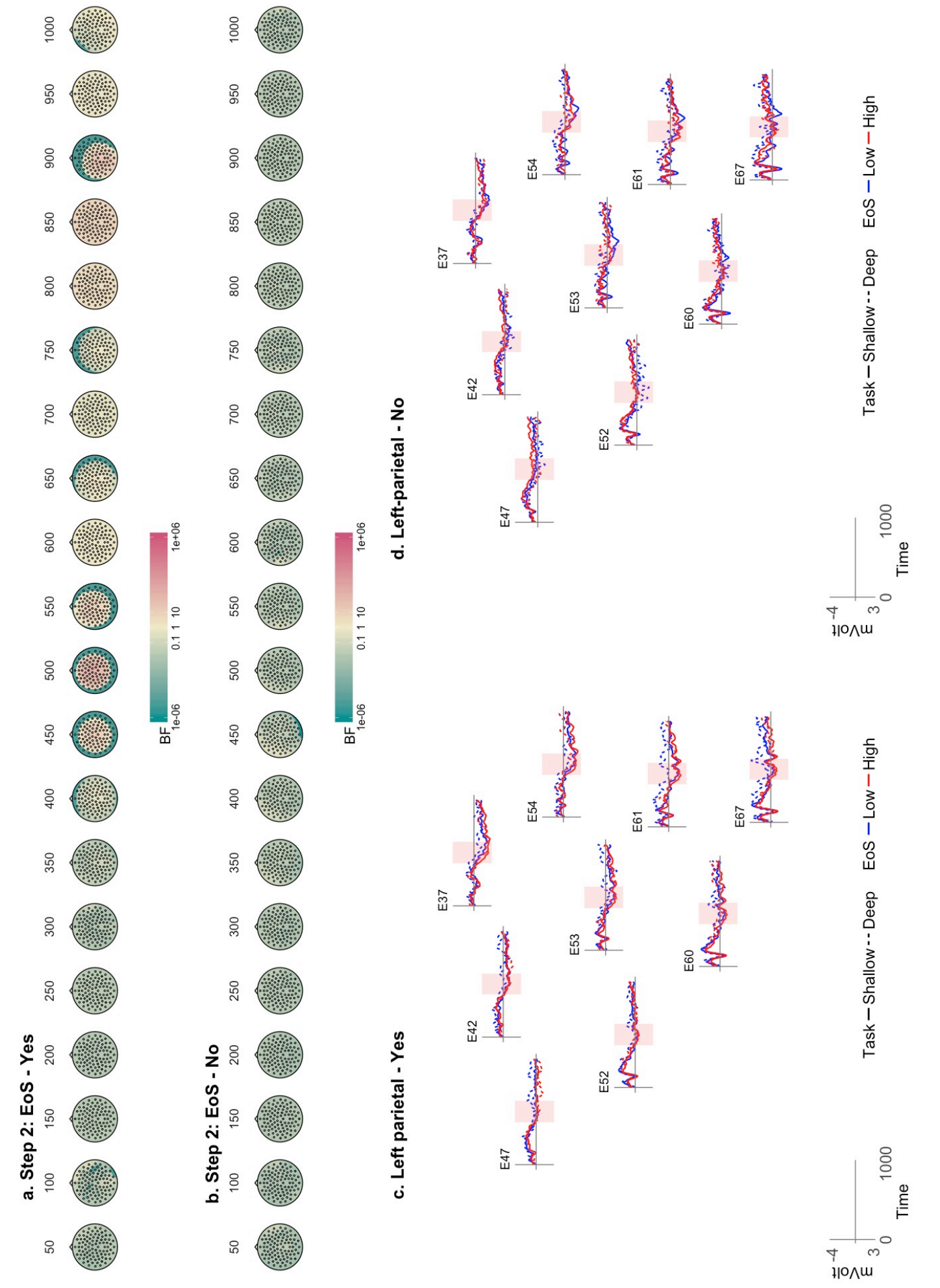

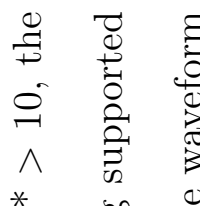

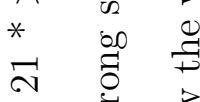

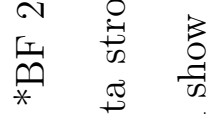

* च च

$3 \stackrel{\Xi}{ \pm}$

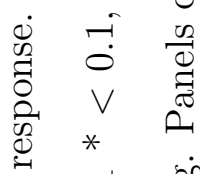

$=\vec{N}$

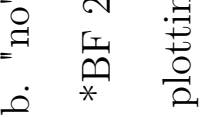

चี

$=0$

๙

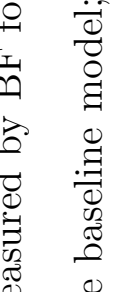

.

भ ष

吕䓃

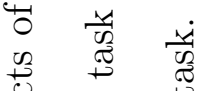

兽劳壳

苨苞苞

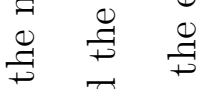

矛苛

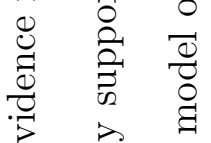

$>$ ह 
Further exploration of EoS's effects found them to go beyond the regions and time windows in the original tentative hypotheses. In fact, EoS had a strong and prolonging main effect across the centre of the scalp when people accepted the metaphors (Figure 6a). The strongest evidence for an EoS effect appeared at E7 in the central area, peaking at the $500 \mathrm{~ms}$ slice $\left(B F_{21}=1925.13 ; b=0.78\right)$. Later in the epoch, EoS again showed a positive effect supported by strong evidence in the parietal area (e.g., E67 at $\left.850 \mathrm{~ms}-900 \mathrm{~ms}, B F_{21}=135.63 ; b=0.79\right)$.

"No" response.

In contrast, the data did not support the effect of EoS on "no" response. The ERP of "no" response was actually better explained by the Step 1 model with task only than the model with task and EoS together (Mean $\left.B F_{21}=0.02 ; \mathrm{SD}=0.05\right)$. That is, on average, the EEG amplitude of rejected trials was 50 times more likely in the task-only model than the model with both task and EoS as predictors. This means that for those metaphors that were eventually rejected as nonsensical or uninterpretable, EoS did not affect the processing within the first $1000 \mathrm{~ms}$.

\section{LDF effects.}

For LDF, our hypotheses stated that we should find evidence for its effect in two areas. In temporal order, first we should find an effect before $350 \mathrm{~ms}$ in the frontal and parietal area, prior to the window when the effect of EoS reached its peak. Second, we should find an effect after $600 \mathrm{~ms}$ to the end of the epoch in the right posterior (i.e., parietal, occipital) regions. We did not find any evidence in Step 2 for LDF's effect, either in these hypothesised regions or on the whole scalp (Figure 7). In fact, we found evidence against the effect of LDF above and beyond the effect of task ("Yes": Mean $B F_{21}=0.02$ (0.08); "No": Mean $B F_{21}=0.03(0.10)$. In other words, the EEG amplitude of the "yes" trials were 50 times more likely, and that of the "no" trials were 33 times more likely, in the task-only models than in 
models including both LDF and task.

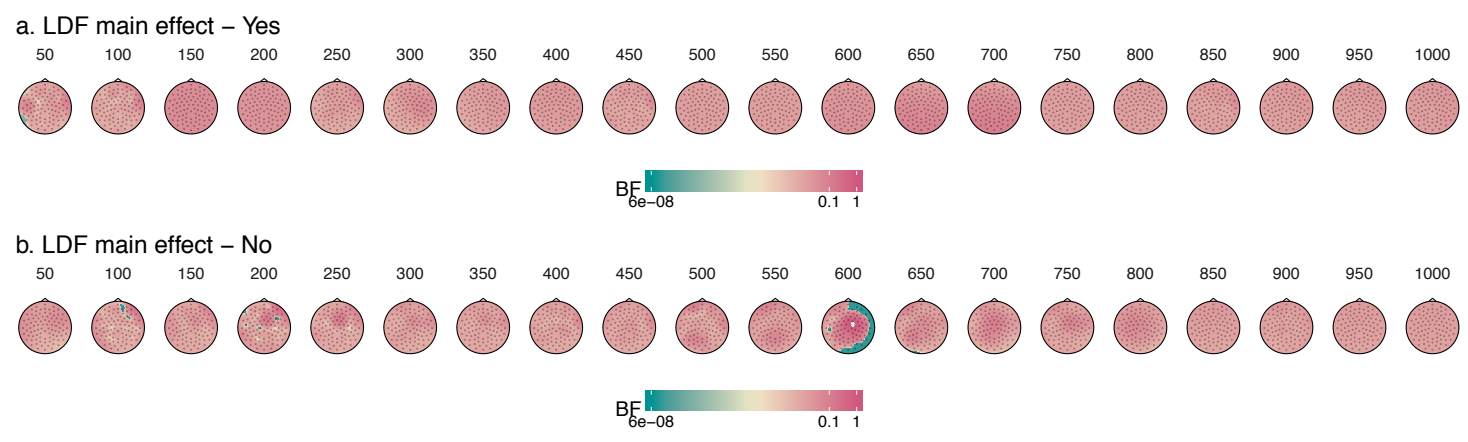

Figure 7. Evidence for main effects of LDF for a. "yes", and b. "no" responses measured by $\mathrm{BF}$. When $\mathrm{BF}<0.1$, the data was strongly against the effect of LDF. BF was logarithmically transformed for plotting.

\section{Moderation effect of the task.}

Step 3 of the hierarchical model comparison dealt specifically with the interaction between task and the key variables. According to our hypotheses, task should interact with both EoS and LDF, though we do not have specific hypotheses concerning the timecourse or the scalp region. It should enhance the effect of EoS in the deep processing task specifically, but enhance the effect of LDF in the shallow processing task instead. To illustrate the differences between shallow and deep tasks, we further analysed the effect of EoS and LDF in separate models per task, which produced event-related regression coefficients (ERRC) which provided the direction and magnitude of effects (Hauk et al., 2009). For example, if the ERRC of EoS was 2.50, it meant that as EoS increased one unit, the EEG amplitude increased for $2.50 \mu \mathrm{V}$.

The interaction effect between EoS and Task was not found in the hypothesised area for "yes" response (i.e., the left parietal area; Figure 8a). Evidence was only found for "no" response (i.e., when people rejected the metaphors), at the end of the epoch in E108 in the right temporal area $\left(B F_{32}=\right.$ 
14.03; Figure 8b), which means that the EEG amplitude of E108 was explained by the model with the interaction between EoS and task 14.03 times better than the model without the interaction. This interaction was driven by a difference between tasks starting from 400ms after the adjective onset (Figure 8c). EoS had a sustaining negative effect in the shallow sensibility judgement task. In other words, at the later stage of processing for sensibility judgement, metaphors that were hard to simulate elicited a greater positivity than those that were easy to simulate; which effect was absent in the deep interpretation generation task (Step 3 model, EoS effect in deep task: $b=0.37$; interaction term: $b=-1.75)$. This difference between tasks culminated at the end of the epoch, thus showing the strong evidence for the interaction model at the final time slice.

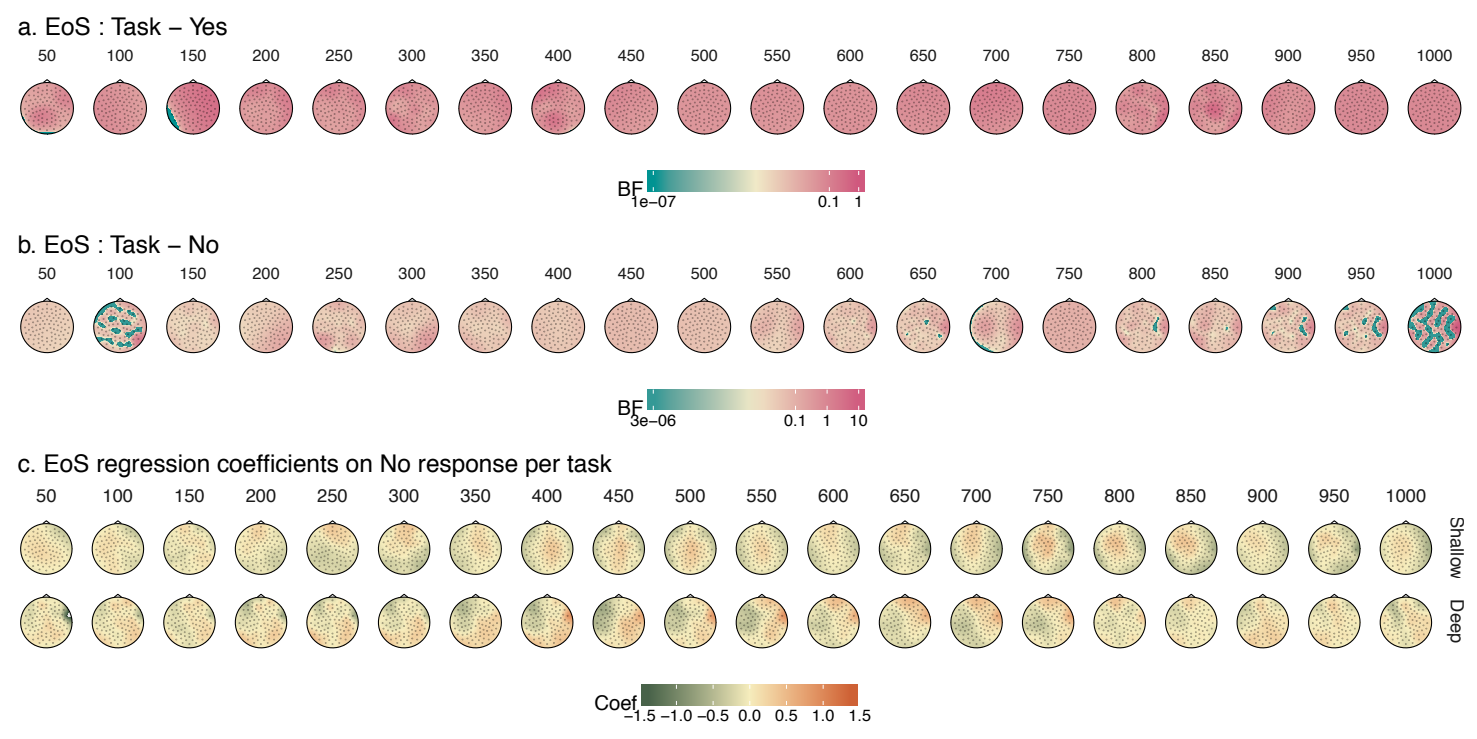

Figure 8. Evidence for the interaction between EoS and task for a. "yes", and b. "no" responses measured by Bayes factors (Step 3 comparison). When $B F_{32}>10$, the data strongly supported the interaction between EoS and task; whereas when $B F_{32}<0.1$, the data strong was against the interaction between EoS task. BF was logarithmically transformed for plotting. Panel c shows the regression coefficients of EoS for "no" response in shallow vs deep processing tasks. 
Interaction between LDF and task was also only found for "no" response. At an early window, evidence was found around $200 \mathrm{~ms}$ at the right frontal $\left(\mathrm{E} 2: B F_{32}=\right.$ 8.49; Figure $9 \mathrm{~b})$ and temporal electrodes (E108: $\left.B F_{32}=7.79\right)$, which means that the data from these two electrodes were about 8 times more likely under models with the interaction between LDF and task than models without the interaction. From Figure 9c, it could be seen that the effect of LDF flipped directions for shallow and deep processing tasks. In E2, LDF had a negative effect on amplitude in the deep processing task (i.e., metaphors whose constituent words rarely co-occurred elicited a greater positivity; LDF in deep task: $b=-1.33$ ) which was smaller and in the opposite direction in the shallow processing task (interaction term: $b=1.44$ ); and in E108, LDF had a positive effect in deep processing (LDF effect in deep task: $b=0.37$; i.e., metaphors whose constituent words rarely co-occurred elicited a greater negativity) but a larger and opposite effect in shallow processing (interaction term: $b=-0.77$; low LDF elicited a greater positivity). This pattern sustained throughout the whole epoch as seen in Figure 9c, but was only supported by Bayes factors between $150 \mathrm{~ms}$ and $250 \mathrm{~ms}$. This early effect in the right frontal region partially supported the hypothesis concerning early effect LDF, although it appeared earlier than the hypothesised time of $350 \mathrm{~ms}$.

Furthermore, we also found some support for the hypothesis that LDF should have a late effect in the right posterior region. At the $750 \mathrm{~ms}$ slice in the right temporal and parietal regions, LDF interacted with task such that the effect of LDF flipped its direction. During deep processing, LDF had a negative effect at E90 in the right parietal region (LDF in deep task: $b=0.51$ ), but a slightly larger, positive effect during shallow processing (interaction term: $b=-1.01$ ). Similar effect was found at E102 in the right temporal electrode (see Supplementary Materials). In other words, metaphors whose constituent words rarely co-occurred elicited a greater positivity in the shallow processing task, but a greater negativity in the deep 
processing task, with the effect on shallow task slightly larger.

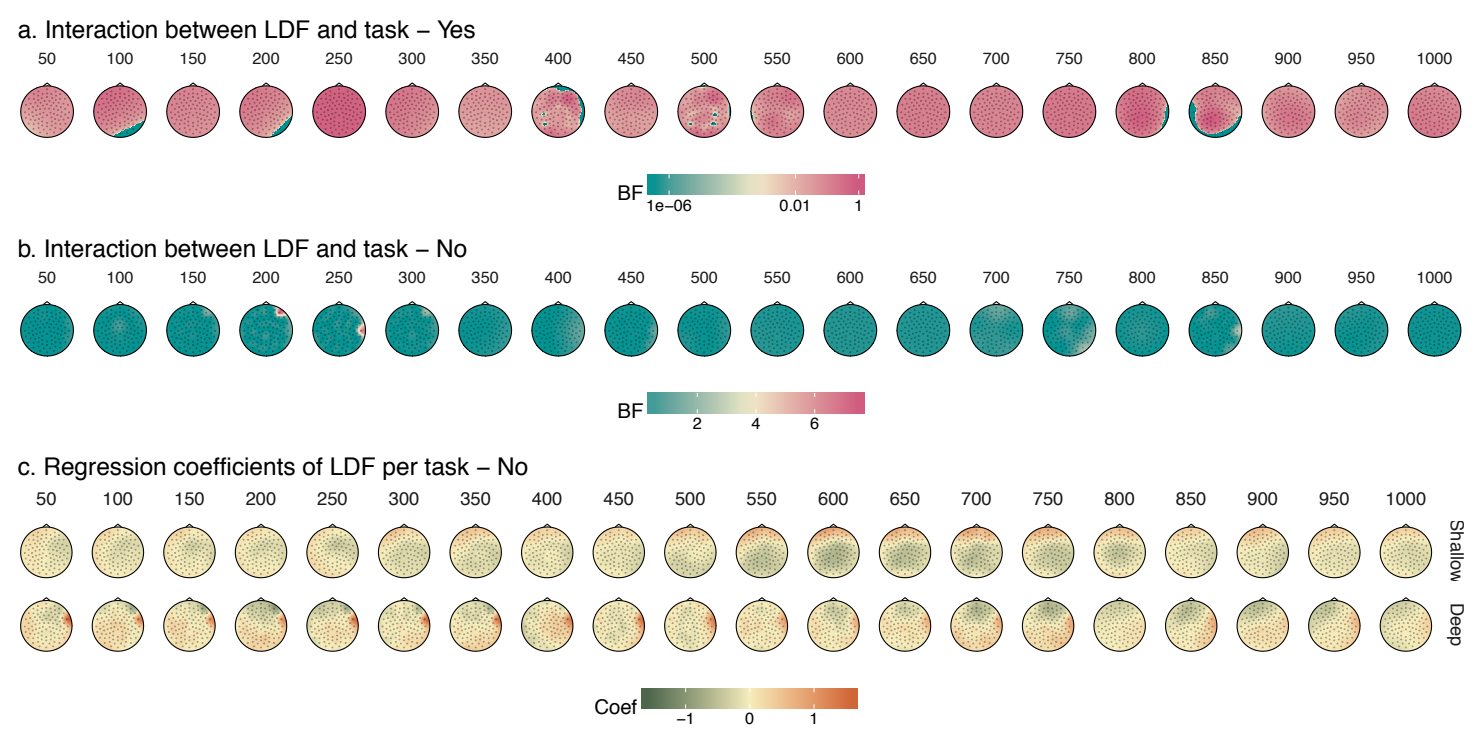

Figure 9. Evidence for the interaction between LDF and task for a. "yes", and b. "no" responses measured by Bayes factors (Step 3 comparison). When $B F_{32}>10$, the data strongly supported the interaction between LDF and task; whereas when $B F_{32}<0.1$, the data strong was against the interaction between LDF task. BF was logarithmically transformed for plotting. Panel c shows the regression coefficients of LDF for "no" response in shallow vs deep processing tasks.

\section{Comparison between EoS and LDF.}

Further model comparisons were carried out to extract information about the relative advantage of EoS and LDF. As we did not find widespread effects of LDF either in Step 2 or in Step 3, here we compared the LDF models obtained in Steps 2 and 3 with the EoS models obtained in Steps 2 and 3 respectively. Such information about the relative strength of EoS and LDF, though both weak at times, was useful for us to pinpoint the timecourse and topography of these variables to see when they differed most strongly.

Figure 10 demonstrated a clear pattern of differences between EoS and LDF. Consistent differences started to emerge after $350 \mathrm{~ms}$ from the adjective onset. While 
EoS mainly accounted for the EEG amplitude in the central region, LDF accounted for the peripheral region. For "yes" response, the largest advantage of LDF over EoS was in the left occipital area (e.g., E71) after $600 \mathrm{~ms}$, similar to our hypothesis that LDF would have a greater effect at a later stage of processing in the posterior region. At $650 \mathrm{~ms}$, the model with $\mathrm{LDF}$ and task was 143 times better than the model with EoS and task at explaining the EEG data $\left(B F_{E L}=0.007\right)$, and the interaction model displayed the same advantage. LDF had a negative effect on the amplitude, which meant that metaphors with less frequently co-occurring words elicited greater positivity than metaphors with more frequently co-occurring words (Step 3 model, LDF in deep task: $b=-0.35$ ), and the effect was larger for shallow processing than deep processing, as predicted by the linguistic shortcut hypothesis. (Step 3 model, interaction term: $b=-0.33$ ).

The largest advantage of LDF over EoS for "no" response is in the right central area at 600ms (see Panels b and d). For example, the data in E106 was 250 times more likely in the model with LDF and task (without interaction) than the model with EoS and task $\left(B F_{E L}=0.004\right)$, and the same advantage was shown when comparing the interaction models too. LDF had, again, a negative effect on the amplitude (i.e, low LDF -> greater positivity; Step 3, LDF in deep task: $b=-0.25$ ), which was larger for shallow processing than deep processing too (Step 3 model, interaction term: $b=-0.38)$.

Summary. The results confirmed that EoS had an effect on EEG amplitude across the centre of the scalp (especially the parietal area) around 400ms when people eventually accepted the metaphors. Although EoS alone did not affect EEG amplitude when people rejected the metaphors, it did interact with task at the end of the 1000ms epoch, suggesting differential activations of the embodied component per task. However, contrary to the hypothesis that EoS would be more involved in the deep interpretation generation task, this specific effect at the end of the epoch 


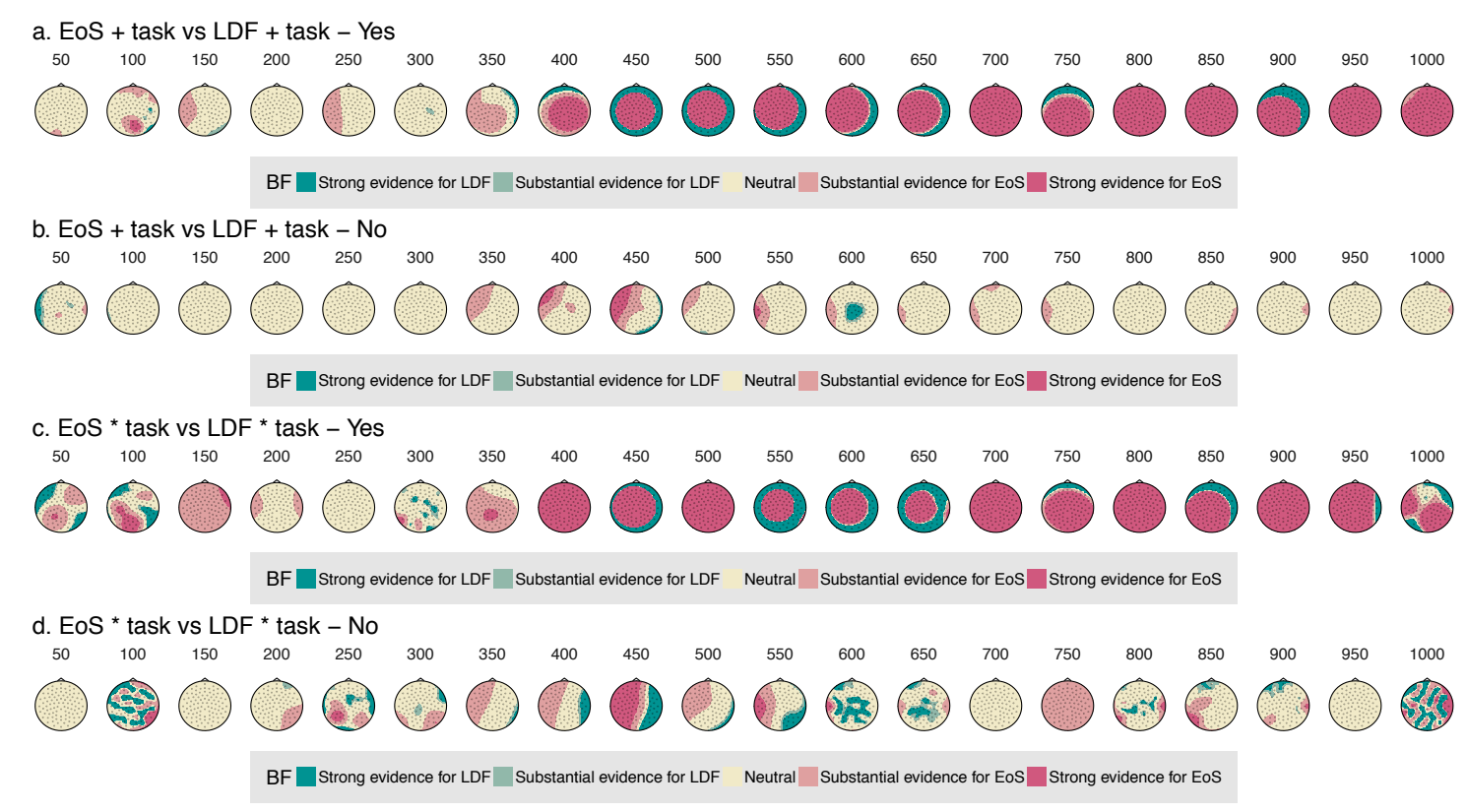

Figure 10. Evidence for EoS compared with evidence for LDF as a result of comparisons of the models obtained from Step 2 (i.e., EoS or LDF and task as fixed predictors; Panels a and b) and Step 3 (i.e., interactions between EoS or LDF and task as fixed predictors; Panels c and d). Strong evidence means that $B F_{E L}>10$ or $B F_{E L}<0.1 ;$ substantial evidence means that $B F_{E L}>3$ or $B F_{E L}<0.33$.

was in fact larger during the shallow sensibility judgement task.

The effect of LDF was only detected for "no" response, which was found to be moderated by task. This effect occurred around 200ms, prior to the effect of EoS, supporting the linguistic shortcut hypothesis. We found that the effect of LDF was in opposite directions for shallow and deep processing tasks, but the magnitude of the effect was not consistently larger during shallow processing. In other words, the evidence showed differential activations of the linguistic component per task, but did not consistently support the hypothesis that the linguistic component was more heavily utiliesd during shallow processing. Evidence for the linguistic shortcut hypothesis showed that the linguistic component had a larger effect during shallow than deep processing at a later stage of processing after $600 \mathrm{~ms}$ from the adjective 
onset.

\section{General Discussion}

The aim of the present study was to examine the nature and timecourse of conceptual representations during metaphor processing. From the behavioural and EEG study, we managed to see how the embodied and linguistic components interplayed to represent the meanings of a metaphor and inform the outcome of processing. Both components were activated within the initial 1000ms of processing, and lasted even until the response was made. Information from the linguistic distributional pattern was initially activated around $200 \mathrm{~ms}$ after the adjective of a metaphor was presented, much earlier than when embodied simulation reached its peak. The effect on EEG approximated the typical P2 effect, usually associated with unexpected visual features of words in context (Federmeier, 2002). This early activation only appeared for metaphors that were eventually rejected as uninterpretable or nonsensical, highlighting the role of the linguistic component in deciding the eventual success/failure of processing. A metaphor that resulted in failed conceptual representation had already been marked out by the linguistic component from as early as $200 \mathrm{~ms}$, when what was generally regarded as semantic processing had not been initiated. It suggested that if the constituent words did not often co-occur, it violated the predictions created by the linguistic distributional patterns, which was informative to the eventual rejection of the metaphor.

After the initial activation of the linguistic components, embodied simulation commenced for those metaphors which were eventually accepted. The temporal difference between embodied and linguistic components was consistent with LASS (Barsalou et al., 2008) and SIH (Louwerse, 2011), which suggested that the linguistic component should reach the peak of its activation before the embodied component. It also satisfied a necessary hypothesis of the linguistic shortcut 
hypothesis, suggesting that the linguistic component can be a quick and dirty guide for embodied component down the line (Connell \& Lynott, 2014). The embodied component was prominent around and after $400 \mathrm{~ms}$, which was consistent with the typical N400 effect, commonly regarded as an indicator of the effort expended on integrating new stimulus into the semantic representation (Kutas \& Federmeier, 2011). This finding offered further support to theories of embodied language comprehension, that is: the so-called semantic processing, a major part of language comprehension, depends on the reactivation of sensory, motor and other bodily experiences (Zwaan, 2004), which was also supported by the previous study on literal language processing (Liu et al., 2018a).

Judging from the neuroelectrical activations of EoS, if the embodied component was activated around 400ms, the metaphors would eventually be accepted. However, for those metaphors that ended up in rejection, the embodied component was not activated until the end of the 1000ms epoch, which suggested that the embodied component might have an effect on the processing of rejected metaphor beyond the initial 1000ms epoch, consistent with the behavioural finding the embodied component affected the rejection speed and rate. The fact that this effect between $950 \mathrm{~ms}$ and 1000ms was moderated by task was also consistent with the behavioural finding that people relied on simulation more during deep processing than shallow processing. It meant that simulation was not or all-or-nothing process, but was subject to the desired depth of processing.

On the other hand, the linguistic component affected the rejection of metaphors again around $750 \mathrm{~ms}$ in the posterior region, as predicted by the tentative hypotheses. Furthermore, this effect was moderated by task too, lending support to the linguistic shortcut hypothesis. These activations, coinciding with the late positivity complex, were often associated with reconciling conflicting representations (Kuperberg, 2007; Paczynski \& Kuperberg, 2012) as well as decision making (Collins 
et al., 2011; Polich, 2007), which will be further discussed in the next section.

\section{Further Discussion Concerning the Linguistic Component}

The role of the linguistic component was of particular interest in this study, which aimed to test whether the linguistic component can act as a shortcut to guide future processing in the embodied component. This hypothesis was supported in two ways. First, the linguistic component was activated before the embodied component was fully engaged. At an early stage of processing, the linguistic component could mark out potentially unsuccessful processing, if the constituent words were unexpected. Second, the effect of the linguistic component, both around $200 \mathrm{~ms}$ and $750 \mathrm{~ms}$, were limited to metaphors that were eventually rejected, which supported the suggestion that one of the functions of the linguistic shortcut was to conserve limited energy if the processing is unlikely to succeed (Connell \& Lynott, 2013).

The effect of the linguistic component was always found to be sensitive to the task demands. It was hypothesised that linguistic component should be more prominent if the task required a detailed, elaborate account of the conceptual representation rather than a simple yes/no decision. Although the ERP results displayed different patterns (i.e., the regression coefficients of LDF at 200ms-250ms was larger for deep processing than shallow processing, but was larger for shallow than deep processing at $700 \mathrm{~ms}-750 \mathrm{~ms}$ ), it did not necessarily mean that the linguistic component was more, or less, activated during deep processing because of the low spatial resolution of EEG. Since the raw waveform displayed the sum of all neural activities in the brain, which for certain included elements other than the activation of linguistic information (Luck, 2005), it was better concluded that the linguistic component was activated differently due to different depths of processing.

According to the behavioural results, the linguistic component could act as a shortcut in two ways (Connell \& Lynott, 2013; Louwerse \& Connell, 2011). One 
possibility is to directly inform the outcome of processing; and the other is to guide the more costly embodied simulation down the line. Both possibilities were found in the current study. First, the linguistic component directly affected the speed to accept a metaphor (both Experiment 1 and Experiment 2), such that the more often two constituent words co-occurred, the easier it was to accept the metaphor as either sensible or interpretable. This behavioural effect was found in spite of the fact that the linguistic component did not affect the processing of accepted metaphors within the first 1000ms epoch, which suggested this function of the linguistic shortcut emerged at a later stage of processing, possibly associated with decision making rather than the semantic processing itself. The linguistic component also directly affected the rejection speed in Experiment 2 but not in Experiment 1. Such a difference between experiments could simply be a result of individuals' various reliance on linguistic information, or the different experiences between a straightforward behavioural study and a long-winded EEG study. However, another possibility, which was of more theoretical importance, suggested that the difference was driven by the 1s delay in response in the EEG study which was not introduced in the behavioural study. The longer response latency could have given participants the opportunity to put more weight on the linguistic linguistic, which was not allowed when participants were required to respond as soon as they saw the metaphors. This suggestion again pointed to the possibility that the direct effect of linguistic shortcut emerged at a later stage of processing from both ERP and behavioural results. Future studies should test this role of the linguistic component specifically.

The second role of the linguistic component was to guide the later, more costly embodied simulation. Again, we found this role to differ in the two experiments. In some cases (such as Experiment 2), the linguistic component provided a signal to reject the metaphor without further processing using embodied simulation, such that 
the embodied component had a smaller effect on metaphors whose constituent words rarely co-occurred (e.g., Illness can be bright) than on those whose constituent words frequently co-occurred (e.g., Supply can be bright; found also in Liu et al., 2017). In other cases (such as Experiment 1), the linguistic component encouraged more effort of processing in the embodied component when two constituent words often co-occurred, such that it became harder to reject the metaphor even when it was difficult to simulate (found also in Liu et al., 2018c). To put it differently, when people saw Supply can be bright, in which case supply and bright often co-occurred in the same context, although they found it hard to simulate the meaning, they were willing to spend more time processing it, and were more reluctant to reject it straight away. In this case, the linguistic shortcut did not perform a triage role to conserve energy, but a safeguarding role to prevent premature rejection of the metaphor.

The process of conceptual representation presented in this study is more complex and dynamic than what current theories suggest. The linguistic component is involved in various stages throughout the processing, possibly exerting its influence from sub-lexical and lexical processing to decision making (Hauk, 2016). The effect of the linguistic component could vary from task to task, and was sensitive to the environment and task demands. However, the present study, together with previous studies (Liu et al., 2017, 2018c), did not find evidence that the linguistic component was more heavily involved when shallow processing could suffice, but that the linguistic component was involved to different degrees, or in different ways, because of the depth of processing. Future studies should consider if these results are unique to metaphor processing, and whether they are due to the way the embodied and linguistic components are operationalised (see detailed discussion in Liu et al., 2018c). 


\section{Conclusion}

In this study, we studied the conceptual representations during metaphor processing. Not only did we confirm previous findings that metaphor processing relied on both embodied and linguistic components, but we also displayed a detailed landscape of the role of the linguistic component and how it interplayed with the embodied component. By studying the timecourse of these components and the effect of task demands, we strongly supported the claim that conceptual representation was task-dependent. The involvement of the linguistic component was extremely flexible and it could affect embodied simulation in numerous ways based on the individual, the environment and the requirement of the task. 


\section{References}

Amsel, B. D. (2011). Tracking real-time neural activation of conceptual knowledge using single-trial event-related potentials. Neuropsychologia, 49(5), 970-983. doi:10.1016/j.neuropsychologia.2011.01.003

Andrews, M., Vigliocco, G., \& Vinson, D. (2009). Integrating experiential and distributional data to learn semantic representations. Psychological Review, 116(3), 463-498. doi:10.1037/a0016261

Arzouan, Y., Goldstein, A., \& Faust, M. (2007). Brainwaves are stethoscopes: ERP correlates of novel metaphor comprehension. Brain Research, 1160, 69-81. doi:10.1016/j.brainres.2007.05.034

Auguie, B. (2017). GridExtra: Miscellaneous functions for "grid" graphics. Retrieved from https://CRAN.R-project.org/package=gridExtra

Aust, F., \& Barth, M. (2017). papaja: Create APA manuscripts with R Markdown. Retrieved from https://github.com/crsh/papaja

Baayen, R. H., Davidson, D., \& Bates, D. (2008). Mixed-effects modeling with crossed random effects for subjects and items. Journal of Memory and Language, 59(4), 390-412. doi:10.1016/J.JML.2007.12.005

Bache, S. M., \& Wickham, H. (2014). Magrittr: A forward-pipe operator for $r$. Retrieved from https://CRAN.R-project.org/package=magrittr

Barr, D. J., Levy, R., Scheepers, C., \& Tily, H. J. (2013). Random effects structure for confirmatory hypothesis testing: Keep it maximal. Journal of Memory and Language, 68(3). doi:10.1016/j.jml.2012.11.001

Barsalou, L. W., Santos, A., Simmons, W. K., \& Wilson, C. (2008). Language and simulation in conceptual processing. In A. M. Glenberg \& A. C. Graesser (Eds.), Symbols, embodiment, and meaning (pp. 245-283). Oxford, England: 
Oxford University Press.

Bates, D., \& Maechler, M. (2017). Matrix: Sparse and dense matrix classes and methods. Retrieved from https://CRAN.R-project.org/package=Matrix

Bates, D., Mächler, M., Bolker, B., \& Walker, S. (2015). Fitting linear mixed-effects models using lme4. Journal of Statistical Software, 67(1), 1-48. doi:10.18637/jss.v067.i01

Brants, T., \& Franz, A. (2006). Web 1T 5-gram Version 1 LDC2006T13. DVD. Philadelphia: Linguistic Data Consortium.

Brouwer, H., Fitz, H., \& Hoeks, J. (2012). Getting real about semantic illusions: rethinking the functional role of the P600 in language comprehension. Brain Research, 1446, 127-43. doi:10.1016/j.brainres.2012.01.055

Clark, H. H. (1973). The language-as-fixed-effect fallacy: A critique of language statistics in psychological research. Journal of Verbal Learning and Verbal Behavior, 12(4), 335-359. doi:10.1016/S0022-5371(73)80014-3

Cohen, J., Cohen, P., West, S. G., \& Aiken, L. S. (2003). Applied Multiple Regression/Correlation Analysis for the Behavioral Sciences. Mahwah, NJ: Erlbaum.

Collins, J., Pecher, D., Zeelenberg, R., \& Coulson, S. (2011). Modality Switching in a Property Verification Task: An ERP Study of What Happens When Candles Flicker after High Heels Click. Frontiers in Psychology, 2(February), 10. doi:10.3389/fpsyg.2011.00010

Connell, L. (2018). What have labels ever done for us? The lingusitic shortcut in conceptual processing. Language, Cognition and Neuroscience. doi:10.1080/23273798.2018.1471512

Connell, L., \& Lynott, D. (2013). Flexible and fast: linguistic shortcut affects both 
shallow and deep conceptual processing. Psychonomic Bulletin \& Review, 20(3), 542-50. doi:10.3758/s13423-012-0368-x

Connell, L., \& Lynott, D. (2014). Principles of representation: Why you can't represent the same concept twice. Topics in Cognitive Science, 6(3), 390-406. doi:10.1111/tops.12097

Coulson, S., \& Van Petten, C. (2002). Conceptual integration and metaphor: An event-related potential study. Memory \& Cognition, 30(6), 958-968. doi:10.3758/BF03195780

Dantzig, S. van, Cowell, R. A., Zeelenberg, R., \& Pecher, D. (2011). A sharp image or a sharp knife: norms for the modality-exclusivity of 774 concept-property items. Behavior Research Methods, 43(1), 145-54. doi:10.3758/s13428-010-0038-8

Davenport, T., \& Coulson, S. (2011). Predictability and novelty in literal language comprehension: an ERP study. Brain Research, 1418, 70-82. doi:10.1016/j.brainres.2011.07.039

De Grauwe, S., Swain, A., \& Holcomb, P. (2010). Electrophysiological insights into the processing of nominal metaphors. Neuropsychologia, 48(7), 1965-1984. doi:10.1016/j.neuropsychologia.2010.03.017.Electrophysiological

Dehaene, S., Naccache, L., Le Clec'H, G., Koechlin, E., Mueller, M., Dehaene-Lambertz, G., ... Le Bihan, D. (1998). Imaging unconscious semantic priming. Nature, 395(6702), 597-600. doi:10.1038/26967

Dowle, M., \& Srinivasan, A. (2017). Data.table: Extension of 'data.frame'. Retrieved from https://CRAN.R-project.org/package=data.table

Federmeier, K. D. (2002). Picture the difference: electrophysiological investigations of picture processing in the two cerebral hemispheres. Neuropsychologia, 
40(7), 730-747. doi:10.1016/S0028-3932(01)00193-2

Gelman, A., \& Su, Y.-S. (2018). Arm: Data analysis using regression and multilevel/hierarchical models. Retrieved from https://CRAN.R-project.org/package=arm

Genz, A., \& Bretz, F. (2009). Computation of multivariate normal and $t$ probabilities. Heidelberg: Springer-Verlag.

Harrell Jr, F. E. (2018). Rms: Regression modeling strategies. Retrieved from https://CRAN.R-project.org/package=rms

Harrell Jr, F. E., Charles Dupont, \& others. (2018). Hmisc: Harrell miscellaneous. Retrieved from https://CRAN.R-project.org/package=Hmisc

Hauk, O. (2016). Only time will tell - why temporal information is essential for our neuroscientific understanding of semantics. Psychonomic Bulletin \& Review, 23(4), 1072-1079. doi:10.3758/s13423-015-0873-9

Hauk, O., Pulvermüller, F., Ford, M., Marslen-Wilson, W. D., \& Davis, M. H. (2009). Can I have a quick word? Early electrophysiological manifestations of psycholinguistic processes revealed by event-related regression analysis of the EEG. Biological Psychology, 80(1), 64-74.

doi:10.1016/j.biopsycho.2008.04.015

Hendrix, P., Bolger, P., \& Baayen, R. H. (2017). Distinct ERP signatures of word frequency, phrase frequency, and prototypicality in speech production. Journal of Experimental Psychology: Learning, Memory, and Cognition, 43(1), 128-149. doi:10.1037/a0040332

Henry, L., \& Wickham, H. (2018). Purrr: Functional programming tools. Retrieved from https://CRAN.R-project.org/package=purrr

Hothorn, T. (2017). TH.data: TH's data archive. Retrieved from 
https://CRAN.R-project.org/package=TH.data

Hothorn, T., Bretz, F., \& Westfall, P. (2008). Simultaneous inference in general parametric models. Biometrical Journal, 50(3), 346-363.

Højsgaard, S., \& Halekoh, U. (2018). DoBy: Groupwise statistics, lsmeans, linear contrasts, utilities. Retrieved from https://CRAN.R-project.org/package=doBy

Jarosz, A. F., \& Wiley, J. (2014). What are the odds? A practical guide to computing and reporting Bayes Factors. The Journal of Problem Solving, 7, 2-9. doi:10.7771/1932-6246.1167

Jeffreys, H. (1998). Theory of probability (3rd ed.). Oxford, UK: Oxford University Press.

Johns, B. T., \& Jones, M. N. (2012). Perceptual Inference Through Global Lexical Similarity. Topics in Cognitive Science, 4(1), 103-120. doi:10.1111/j.1756-8765.2011.01176.x

Koenker, R., \& Ng, P. (2017). SparseM: Sparse linear algebra. Retrieved from https://CRAN.R-project.org/package=SparseM

Kuhn, M., Steve Weston, Wing, J., Forester, J., \& Thaler, T. (2016). Contrast: A collection of contrast methods. Retrieved from https://CRAN.R-project.org/package=contrast

Kuperberg, G. R. (2007). Neural mechanisms of language comprehension:

Challenges to syntax. Brain Research, 1146(1), 23-49. doi:10.1016/j.brainres.2006.12.063

Kutas, M., \& Federmeier, K. D. (2011). Thirty years and counting: finding meaning in the N400 component of the event-related brain potential (ERP). Annual 
Review of Psychology, 62, 621-47. doi:10.1146/annurev.psych.093008.131123

Kutas, M., Federmeier, K. D., \& Sereno, M. I. (1999). Current approaches to mapping language in electromagnetic space. In C. Brown \& P. Hagoort (Eds.), The neurocognition of language (pp. 359-392). New York: Oxford University Press. doi:10.1093/acprof:oso/9780198507932.003.0012

Kuznetsova, A., Brockhoff, P. B., \& Christensen, R. H. B. (2017). lmerTest package: Tests in linear mixed effects models. Journal of Statistical Software, 82(13), 1-26. doi:10.18637/jss.v082.i13

Lebois, L. A. M., Wilson-Mendenhall, C. D., \& Barsalou, L. W. (2015). Are Automatic Conceptual Cores the Gold Standard of Semantic Processing? The Context-Dependence of Spatial Meaning in Grounded Congruency Effects. Cognitive Science, 39(8), 1764-1801. doi:10.1111/cogs.12174

Liu, P. Q., Connell, L., \& Lynott, D. (2017). Can illness be bright? Metaphor comprehension depends on linguistic and embodied factors. In G. Gunzelmann, A. Hows, T. Tenbrink, \& E. J. Davelaar (Eds.), 39th annual conference of cognitive science society (pp. 2604-2609). Austin, TX: Cognitive Science Society.

Liu, P. Q., Connell, L., \& Lynott, D. (2018a). Continuous neural activities of modality switching costs. Manuscript in Preparation.

Liu, P. Q., Connell, L., \& Lynott, D. (2018b). Ease-of-Simulation Norms for 452 Adjective Metaphors. Manuscript Under Review, 1-27.

Liu, P. Q., Connell, L., \& Lynott, D. (2018c). Effect of time constraints on the grounded representation of metaphor processing. Manuscript in Preparation, $1-30$.

Louwerse, M. M. (2011). Symbol interdependency in symbolic and embodied cognition. Topics in Cognitive Science, 3(2), 273-302. 
doi:10.1111/j.1756-8765.2010.01106.x

Louwerse, M. M., \& Connell, L. (2011). A taste of words: linguistic context and perceptual simulation predict the modality of words. Cognitive Science, 35(2), 381-98. doi:10.1111/j.1551-6709.2010.01157.x

Louwerse, M. M., \& Hutchinson, S. (2012). Neurological evidence linguistic processes precede perceptual simulation in conceptual processing. Frontiers in Psychology, 3(October), 385. doi:10.3389/fpsyg.2012.00385

Louwerse, M. M., \& Jeuniaux, P. (2010). The linguistic and embodied nature of conceptual processing. Cognition, 114(1), 96-104. doi:10.1016/j.cognition.2009.09.002

Luck, S. J. (2005). Ten Simple Rules for Deisgning ERP Experiments. In Event-related potentials: A methods handbook (pp. 17-32). doi:10.1371/journal.pcbi.0020012

Lynott, D., \& Connell, L. (2009). Modality exclusivity norms for 423 object properties. Behavior Research Methods, 41(2), 558-64. doi:10.3758/BRM.41.2.558

Lynott, D., \& Connell, L. (2010). Embodied conceptual combination. Frontiers in Psychology, 1(November), 212. doi:10.3389/fpsyg.2010.00212

Morey, R. D., \& Rouder, J. N. (2015). BayesFactor: Computation of bayes factors for common designs. Retrieved from https://CRAN.R-project.org/package=BayesFactor

Nowosad, J. (2017). Rcartocolor: 'CARTOColors' palettes. Retrieved from https://CRAN.R-project.org/package=rcartocolor

Paczynski, M., \& Kuperberg, G. R. (2012). Multiple influences of semantic memory on sentence processing: Distinct effects of semantic relatedness on violations 
of real-world event/state knowledge and animacy selection restrictions. Journal of Memory and Language, 67(4), 426-448. doi:10.1016/j.jml.2012.07.003

Pinheiro, J., Bates, D., DebRoy, S., Sarkar, D., \& R Core Team. (2017). nlme: Linear and nonlinear mixed effects models. Retrieved from https://CRAN.R-project.org/package=nlme

Plate, T., \& Heiberger, R. (2016). Abind: Combine multidimensional arrays. Retrieved from https://CRAN.R-project.org/package=abind

Plummer, M., Best, N., Cowles, K., \& Vines, K. (2006). CODA: Convergence diagnosis and output analysis for mcmc. R News, 6(1), 7-11. Retrieved from https://journal.r-project.org/archive/

Polich, J. (2007). Updating P300: an integrative theory of P3a and P3b. Clinical Neurophysiology : Official Journal of the International Federation of Clinical Neurophysiology, 118(10), 2128-48. doi:10.1016/j.clinph.2007.04.019

Pynte, J., Besson, M., Robichon, F., \& Poli, J. (1996). The time-course of metaphor comprehension: An event-related potential study. Brain and Language, 316(55), 293-316.

R Core Team. (2017). R: A language and environment for statistical computing. Vienna, Austria: R Foundation for Statistical Computing. Retrieved from https://www.R-project.org/

Revelle, W. (2017). Psych: Procedures for psychological, psychometric, and personality research. Evanston, Illinois: Northwestern University. Retrieved from https://CRAN.R-project.org/package=psych

Robinson, D. (2018). Broom: Convert statistical analysis objects into tidy data 
frames. Retrieved from https://CRAN.R-project.org/package=broom

Sarkar, D. (2008). Lattice: Multivariate data visualization with r. New York: Springer. Retrieved from http://lmdvr.r-forge.r-project.org

Solt, F., \& Hu, Y. (2015). interplot: Plot the effects of variables in interaction terms. Available at The Comprehensive R Archive Network (CRAN). Retrieved from https://CRAN.R-project.org/package=interplot

Terry M. Therneau, \& Patricia M. Grambsch. (2000). Modeling survival data: Extending the Cox model. New York: Springer.

Venables, W. N., \& Ripley, B. D. (2002). Modern applied statistics with s (Fourth.). New York: Springer. Retrieved from http://www.stats.ox.ac.uk/pub/MASS4

Vigliocco, G., Meteyard, L., Andrews, M., \& Kousta, S. (2009). Toward a theory of semantic representation. Language and Cognition, 1(2), 219-247. doi:10.1515/LANGCOG.2009.011

Wagenmakers, E.-J. (2007). A practical solution to the pervasive problems of $\mathrm{p}$ values. Psychonomic Bulletin \& Review, 14(5), 779-804. doi:10.3758/BF03194105

Wickham, H. (2009). Ggplot2: Elegant graphics for data analysis. Springer-Verlag New York. Retrieved from http://ggplot2.org

Wickham, H. (2011). The split-apply-combine strategy for data analysis. Journal of Statistical Software, 40(1), 1-29. Retrieved from http://www.jstatsoft.org/v40/i01/

Wickham, H., \& Henry, L. (2017). Tidyr: Easily tidy data with 'spread()' and 'gather()' functions. Retrieved from https://CRAN.R-project.org/package=tidyr

Wickham, H., François, R., Henry, L., \& Müller, K. (2018). Dplyr: A grammar of 
data manipulation. Retrieved from

https://CRAN.R-project.org/package=dplyr

Wilke, C. O. (2017). Cowplot: Streamlined plot theme and plot annotations for 'ggplot2'. Retrieved from https://CRAN.R-project.org/package=cowplot

Wood, S. N. (2003). Thin-plate regression splines. Journal of the Royal Statistical Society (B), 65(1), 95-114.

Wood, S. N. (2004). Stable and efficient multiple smoothing parameter estimation for generalized additive models. Journal of the American Statistical Association, 99(467), 673-686.

Wood, S. N. (2011). Fast stable restricted maximum likelihood and marginal likelihood estimation of semiparametric generalized linear models. Journal of the Royal Statistical Society (B), 73(1), 3-36.

Wood, S., N., Pya, \& S"afken, B. (2016). Smoothing parameter and model selection for general smooth models (with discussion). Journal of the American Statistical Association, 111, 1548-1575.

Xie, Y. (2015). Dynamic documents with $R$ and knitr (2nd ed.). Boca Raton, Florida: Chapman; Hall/CRC. Retrieved from https://yihui.name/knitr/

Xie, Y. (2016). Bookdown: Authoring books and technical documents with $R$ markdown. Boca Raton, Florida: Chapman; Hall/CRC. Retrieved from https://github.com/rstudio/bookdown

Zeileis, A., \& Croissant, Y. (2010). Extended model formulas in R: Multiple parts and multiple responses. Journal of Statistical Software, 34(1), 1-13. doi:10.18637/jss.v034.i01

Zwaan, R. A. (2004). The immersed experiences: toward an embodied theory of language comprehension. In B. H. Ross (Ed.), The psychology of learning and 
motivation (Vol. 44, pp. 35-62). New York, NY, US: Elseview Science.

Zwaan, R. A. (2014). Embodiment and language comprehension: Reframing the discussion. Trends in Cognitive Sciences, 18(5), 229-234. doi:10.1016/j.tics.2014.02.008 